\title{
Trkalian fields and Radon transformation
}

\author{
K. Saygil用 \\ Department of Mathematics, Yeditepe University, Kayisdagi, 34755 Istanbul, Turkey
}

\begin{abstract}
We write the spherical curl transformation for Trkalian fields using differential forms. Then we consider Radon transform of these fields. The Radon transform of a Trkalian field satisfies a corresponding eigenvalue equation on a sphere in transform space. The field can be reconstructed using knowledge of the Radon transform on a canonical hemisphere. We consider relation of the Radon transformation with Biot-Savart integral operator and discuss its transform introducing Radon-BiotSavart operator. The Radon transform of a Trkalian field is an eigenvector of this operator. We also present an Ampere law type relation for these fields. We apply these to Lundquist solution. We present a Chandrasekhar-Kendall type solution of the corresponding equation in the transform space. Lastly, we focus on the Euclidean topologically massive Abelian gauge theory. The Radon transform of an anti-self-dual field is related by antipodal map on this sphere to the transform of the self-dual field obtained by inverting space coordinates. The Lundquist solution provides an example of quantization of topological mass in this context.
\end{abstract}

\section{INTRODUCTION}

The eigenvectors of the curl operator are called Beltrami fields. These are specifically called Trkalian if the eigenvalue is constant. These arises in different areas ranging from fluid dynamics and plasma physics to field theories.

The field theoretic examples of Trkalian vectors are the Euclidean topologically massive $\underline{\underline{1}} \underline{\underline{6}}$ Abelian gauge fields and force-free ${ }^{7}$ magnetic fields. The topologically massive gauge theories $\underline{\underline{1}} \underline{\underline{3}}$ are qualitatively different from Yang-Mills type gauge theories besides their mathematical elegance and consistency. In this context, Trkalian type solutions on 3 -sphere $\mathbb{S}^{3}$, anti-de Sitter space $\mathbb{H}^{3}$ and other spaces in connection with contact geometry are discussed in Refs. $4-6$.

The Curl transformation, as an interesting tool, is introduced in Ref. 8. The (spherical) curl transformation is developed for force-free magnetic fields using vectors $\stackrel{9.10}{~ T h e ~ s p h e r i c a l ~ c u r l ~ t r a n s f o r m ~ a n d ~ R a d o n ~ t r a n s f o r m ~ a r e ~}$ equivalent descriptions. $\stackrel{10}{\underline{0}}$ More precisely, the spherical curl transformation is a Radon probe transformation $\stackrel{11}{\underline{11}}$ Then the spherical curl transformation is applied to the Euclidean topologically massive Abelian gauge theory on $\mathbb{R}^{3}$ in Ref. 6. A complex spinor formalism is also developed in Refs. 12, 13.

The Radon transformation and other variants of it have provided valuable insight into problems in different areas ranging from tomography to twistor theory. The Radon transformation can be defined for differential forms of certain orders in various dimensions $\underline{14,15}$

In the next section, we shall first write the spherical curl transform for Trkalian fields using differential forms. Then we shall discuss Radon transform of these fields in its own right. The Radon transform of a Trkalian field satisfies a corresponding eigenvalue equation on a sphere in transform space whose radius is determined by the eigenvalue. This correspondence can also be inverted. The field can be reconstructed using knowledge of the Radon transform on a canonical hemisphere $\underline{\underline{16}}$

In Section [II, we shall consider the relation of the Radon transformation with Biot-Savart 17 ( $B S)$ integral operator. We shall also present an Ampere law type relation 4.5 for the Trkalian fields. Then we shall discuss Radon transform of the $B S$ integral introducing Radon-Biot-Savart $(R B S)$ operator in the transform space. The Radon transform of a Trkalian field is an eigenvector of the $R B S$ operator.

In Section IV, we shall first present applications of our constructions on Lundquist $\frac{18}{18}$ (L) solution. Then we shall briefly present Chandrasekhar-Kendall 19 (CK) method with circular and elliptic cylindrical solutions. We shall make use of an analogous method for finding solutions of the corresponding equation in the transform space. This yields an eigenvector of the $R B S$ operator.

The Trkalian fields yield interesting solutions of not only the topologically massive gauge theories $\underline{-6}-\underline{6}$ but gravity 20 as well. We shall focus on the Euclidean topologically massive Abelian gauge theory in Section $\mathrm{V}$ The Radon transform of an anti-self-dual potential (or field) is related by antipodal map on the sphere to the transform of the self-dual potential obtained by inverting space coordinates.

To the knowledge of the author, L and CK solutions have been overlooked in the context of topologically massive gauge theories. The L solution provides an example of quantization of the topological mass ${ }^{1-4,6}$ in this context.

*Electronic address: ksaygili@yeditepe.edu.tr 
We provide the necessary definitions and proofs in an appendix.

\section{THE RADON TRANSFORMATION OF TRKALIAN FIELDS}

\section{A. The spherical curl transformation}

We shall write the spherical curl transformation using differential forms in this subsection. The formulation in differential forms follows the same line of reasoning with formally analogous manipulations as given for vectors ${ }^{8}-10$

\section{The Moses coframe and the eigenbasis}

We use the usual correspondence between vectors and 1-forms: $\boldsymbol{Q}_{\lambda}(\boldsymbol{k}) \leftrightarrow \omega^{a}(\boldsymbol{k})$ on $\mathbb{R}^{3}$. We shall also make use of the correspondences $\nabla \times \leftrightarrow * d, \nabla \cdot \leftrightarrow d *$, dot product: $(\cdot) \leftrightarrow(\wedge *)$, cross product: $(\times) \leftrightarrow *(\wedge)$ between basic operations on vectors and differential forms.

The $\operatorname{Moses}^{8}$ eigenbasis 1 -forms

$$
\chi^{a}(\boldsymbol{x} \mid \boldsymbol{k})=\frac{1}{(2 \pi)^{3 / 2}} e^{i \boldsymbol{k} \cdot \boldsymbol{x}} \omega^{a}(\boldsymbol{k}),
$$

of operator $* d$

$$
\begin{aligned}
& * d \chi^{a}(\boldsymbol{x} \mid \boldsymbol{k})=\lambda k \chi^{a}(\boldsymbol{x} \mid \boldsymbol{k}), \\
& d * \chi^{a}(\boldsymbol{x} \mid \boldsymbol{k})=0, \quad a=1,2, \\
& d * \chi^{3}(\boldsymbol{x} \mid \boldsymbol{k})=-\frac{1}{(2 \pi)^{3 / 2}} i k e^{i \boldsymbol{k} \cdot \boldsymbol{x}} * 1,
\end{aligned}
$$

form an orthogonal and complete set

$$
\begin{aligned}
& \left(\chi^{b}\left(\boldsymbol{x} \mid \boldsymbol{k}^{\prime}\right), \chi^{a}(\boldsymbol{x} \mid \boldsymbol{k})\right)=\int \chi^{a}(\boldsymbol{x} \mid \boldsymbol{k}) \wedge \bar{\star} \chi^{b}\left(\boldsymbol{x} \mid \boldsymbol{k}^{\prime}\right)=\delta^{a b} \delta\left(\boldsymbol{k}-\boldsymbol{k}^{\prime}\right) \\
& \sum_{a} \int\left|\chi^{a}(\boldsymbol{x} \mid \boldsymbol{k})><\chi^{a}\left(\boldsymbol{x}^{\prime} \mid \boldsymbol{k}\right)\right| d^{3} k=I \delta\left(\boldsymbol{x}-\boldsymbol{x}^{\prime}\right)
\end{aligned}
$$

Here we use inner product and interior product (bra-ket) notation for 1-forms.

The complex-valued Moses coframe

$$
\begin{aligned}
& \omega^{a}(\boldsymbol{k})=-\frac{\lambda}{\sqrt{2}}\left\{\left[\frac{k_{1}\left(k_{1}+i \lambda k_{2}\right)}{k\left(k+k_{3}\right)}-1\right] d x^{1}+\left[\frac{k_{2}\left(k_{1}+i \lambda k_{2}\right)}{k\left(k+k_{3}\right)}-i \lambda\right] d x^{2}+\frac{k_{1}+i \lambda k_{2}}{k} d x^{3}\right\} \\
& \quad a=1: \lambda=1, a=2: \lambda=-1, \\
& \omega^{3}(\boldsymbol{k})=-\frac{\tilde{k}}{k}=-\kappa, \quad \tilde{k}=k_{1} d x^{1}+k_{2} d x^{2}+k_{3} d x^{3}, \quad k=|\boldsymbol{k}|,
\end{aligned}
$$

is dual to the basis $\left\{\boldsymbol{Q}_{\lambda}(\boldsymbol{k})\right\}$ in Fourier space. ${ }^{8}$ The helicity states are given as $a=1: \lambda=1, a=2: \lambda=-1$, $a=3: \lambda=0$ with our conventions. The basis 1-forms of the Moses coframe respectively satisfy the orthogonality and completeness relations

$$
\begin{aligned}
& \omega^{a}(\boldsymbol{k}) \wedge * \bar{\omega}^{b}(\boldsymbol{k})=<\omega^{b}(\boldsymbol{k}), \omega^{a}(\boldsymbol{k})>* 1, \\
& \sum_{a}\left|\omega^{a}(\boldsymbol{k})><\omega^{a}(\boldsymbol{k})\right|=I, \quad I=\left(\delta_{i j}\right) .
\end{aligned}
$$

The coframe endows $\mathbb{R}^{3}$ with the standard metric 


$$
\begin{aligned}
d s^{2} & =\eta_{a b} \omega^{a} \omega^{b} \\
& =g_{i j} d x^{i} d x^{j},
\end{aligned}
$$

where

$$
\left(\eta_{a b}\right)=\left(\begin{array}{ccc}
0 & -1 & 0 \\
-1 & 0 & 0 \\
0 & 0 & 1
\end{array}\right), \quad\left(g_{i j}\right)=\left(\delta_{i j}\right)
$$

We can easily write the following relations

$$
\begin{array}{ll}
\omega^{1}(\boldsymbol{k})=-\bar{\omega}^{2}(\boldsymbol{k}), \quad \omega^{2}(\boldsymbol{k})=-\bar{\omega}^{1}(\boldsymbol{k}), \quad \omega^{3}(\boldsymbol{k})=\bar{\omega}^{3}(\boldsymbol{k}) \\
\omega^{a}(\boldsymbol{k})=\omega^{a}(\boldsymbol{\kappa}), \quad \boldsymbol{\kappa}=\boldsymbol{k} / k, & \\
\omega^{a}(-\boldsymbol{\kappa})=-\frac{\kappa_{1}+i \lambda \kappa_{2}}{\kappa_{1}-i \lambda \kappa_{2}} \bar{\omega}^{a}(\boldsymbol{\kappa}), & a=1: \lambda=1, a=2: \lambda=-1, \\
\omega^{3}(\boldsymbol{k}) \wedge \omega^{a}(\boldsymbol{k})=i \lambda * \omega^{a}(\boldsymbol{k}), & a=1: \lambda=1, a=2: \lambda=-1, a=3: \lambda=0 \\
\omega^{a}(\boldsymbol{k}) \wedge \bar{\omega}^{b}(\boldsymbol{k})=i \lambda \delta^{a b} * \omega^{3}(\boldsymbol{k}), & a, b=1: \lambda=1, a, b=2: \lambda=-1, \\
\omega^{3}(\boldsymbol{k}) \wedge * \omega^{a}(\boldsymbol{k})=0, & a=1,2, \\
\omega^{3}(\boldsymbol{k}) \wedge * \omega^{3}(\boldsymbol{k})=* 1, & \\
\omega^{1}(\boldsymbol{\kappa}) \wedge \omega^{2}(\boldsymbol{\kappa}) \wedge \omega^{3}(\boldsymbol{\kappa})=-i * 1 . &
\end{array}
$$

Here $\left(^{-}\right)$denotes complex conjugation, (*) is the Hodge dual, $* 1=d x^{1} \wedge d x^{2} \wedge d x^{3}, \omega=\left|\omega_{i}^{a}(\boldsymbol{\kappa})\right|=i, \sqrt{|\eta|}=i, \epsilon_{123}=1$ and $* *=1$. We refer the reader to Refs. $8-10,21,22$ for a comparison with formulation in vectors.

\section{Trkalian field}

The curl transform of a 1-form field is given by an expansion in terms of these eigenforms as in the vectorial case. We can think of this as a plane wave expansion in the coframe $\left\{\omega^{a}\right\}$.

A Trkalian field $* F$

$$
* d * F-\nu * F=0
$$

can be expressed as

$$
* F(\boldsymbol{x})=\sum_{a}^{\prime} *^{a} F(\boldsymbol{x}),
$$

where

$$
*^{a} F(\boldsymbol{x})=\frac{1}{g} \int \chi^{a}(\boldsymbol{x} \mid \boldsymbol{k}) f_{a}(\boldsymbol{k}) d^{3} k,
$$

excluding the divergenceful component $\stackrel{6.9 .10}{ }$ The factor $1 / g$ is introduced for the sake of a proper strength for the gauge potential in topologically massive gauge theory $\underline{\underline{6}}$ This can be taken as 1 for general Trkalian fields. Then the curl transform of $*^{a} F(\boldsymbol{x})$ is given as

$$
f_{a}(\boldsymbol{k})=g\left(\chi^{a}(\boldsymbol{x} \mid \boldsymbol{k}), * F(\boldsymbol{x})\right) .
$$


We find

$$
f_{a}(\boldsymbol{k})=\frac{\delta(k-\lambda \nu)}{k^{2}} s_{a}(\boldsymbol{k})
$$

using a radial delta function. ${ }^{9}$ An arbitrary solution is given entirely in terms of its transform on the sphere of radius $k=\lambda \nu=|\nu|$. Furthermore, only the eigenforms for which $\lambda=\operatorname{sgn}(\nu)$ contribute to the field (12) $: \frac{9}{?}$ The expansion (12) simplifies into

$$
\begin{aligned}
*^{a} F(\boldsymbol{x}) & =\frac{1}{g} \int \chi^{a}(\boldsymbol{x} \mid \lambda \nu \boldsymbol{\kappa}) s_{a}(\lambda \nu \boldsymbol{\kappa}) d \Omega \\
& =\frac{1}{(2 \pi)^{3 / 2}} \frac{1}{g} \int e^{i \lambda \nu \boldsymbol{\kappa} \cdot \boldsymbol{x}} \omega^{a}(\boldsymbol{\kappa}) s_{a}(\lambda \nu \boldsymbol{\kappa}) d \Omega,
\end{aligned}
$$

where $d \Omega$ is the spherical area element and $\boldsymbol{\kappa}=\boldsymbol{k} / k$ is a unit vector in transform space. Therefore a solution can be defined entirely by the value of its curl transform on the unit sphere in transform space $\underline{\underline{9}}$ We call $s_{a}$ the spherical curl transform 10 in order to distinguish it from the full curl transform $f_{a}$.

The spherical curl transform $\frac{10}{\underline{10}}$ is given as

$$
s_{a}(\lambda \nu \boldsymbol{\kappa})=\frac{1}{(2 \pi)^{1 / 2}} g \nu^{2} e^{-i \lambda \nu p} F_{a}^{\mathcal{R}}(p, \boldsymbol{\kappa}),
$$

where

$$
\begin{aligned}
F_{a}^{\mathcal{R}}(p, \boldsymbol{\kappa}) & =\int *{ }^{a} F(\boldsymbol{x}) \wedge \bar{*} \omega^{a}(\boldsymbol{\kappa}) \delta(p-\boldsymbol{\kappa} \cdot \boldsymbol{x}) \\
& =<\omega^{a}(\boldsymbol{\kappa}), \int *^{a} F(\boldsymbol{x})>\delta(p-\boldsymbol{\kappa} \cdot \boldsymbol{x}) * 1,
\end{aligned}
$$

is the Radon transform of the component $F_{a}(\boldsymbol{x})=<\omega^{a}(\boldsymbol{\kappa}), *^{a} F(\boldsymbol{x})>$ of $*{ }^{a} F(\boldsymbol{x})$. The Radon transform of a function (see Appendix A1) is basically defined as the integral of that function over the plane at a distance $p=\boldsymbol{\kappa} \cdot \boldsymbol{x}$ to the origin with unit normal $\kappa$.

We find

$$
F_{a}^{\mathcal{R}}(p, \boldsymbol{\kappa})=(2 \pi)^{1 / 2} \frac{1}{g} \frac{1}{\nu^{2}}<\omega^{a}(\boldsymbol{\kappa}),\left[e^{i \lambda \nu p} \omega^{a}(\boldsymbol{\kappa}) s_{a}(\lambda \nu \boldsymbol{\kappa})+e^{-i \lambda \nu p} \omega^{a}(-\boldsymbol{\kappa}) s_{a}(-\lambda \nu \boldsymbol{\kappa})\right]>,
$$

substituting (16) in (18) and following the reasoning in Ref. 10 with our notation. We shall not repeat these calculations here since they are formally analogous. Then we construct

$$
*{ }^{a} F^{\mathcal{R}}(p, \boldsymbol{\kappa})=(2 \pi)^{1 / 2} \frac{1}{g} \frac{1}{\nu^{2}}\left[e^{i \lambda \nu p} \omega^{a}(\boldsymbol{\kappa}) s_{a}(\lambda \nu \boldsymbol{\kappa})+e^{-i \lambda \nu p} \omega^{a}(-\boldsymbol{\kappa}) s_{a}(-\lambda \nu \boldsymbol{\kappa})\right],
$$

using the completeness relation (5). We can write the spherical curl transform (17) as

$$
\begin{aligned}
s_{a}(\lambda \nu \boldsymbol{\kappa}) & =\frac{1}{(2 \pi)^{1 / 2}} g \nu^{2} e^{-i \lambda \nu p} *\left[*^{a} F^{\mathcal{R}}(p, \boldsymbol{\kappa}) \wedge \bar{*} \omega^{a}(\boldsymbol{\kappa})\right] \\
& =\frac{1}{(2 \pi)^{1 / 2}} g \nu^{2} e^{-i \lambda \nu p}<\omega^{a}(\boldsymbol{\kappa}), *^{a} F^{\mathcal{R}}(p, \boldsymbol{\kappa})>.
\end{aligned}
$$

This is a Radon probe transformation 11 (see Appendix A1). The Radon transform $*^{a} F^{R}(p, \boldsymbol{\kappa})$ is composed of both helicity components of the field $\stackrel{10}{\underline{0}}$ This satisfies the relations

$$
*{ }^{a} F^{\mathcal{R}}(-p,-\boldsymbol{\kappa})=*{ }^{a} F^{\mathcal{R}}(p, \boldsymbol{\kappa}), \quad *{ }^{a} F^{\mathcal{R}}(-p, \boldsymbol{\kappa})=*{ }^{a} F^{\mathcal{R}}(p,-\boldsymbol{\kappa}) .
$$

See Appendix B for equivalence of the spherical curl and Radon transformations.

We have given a formulation of the spherical curl transformation in terms of differential forms. We shall use vector formulation below which is more suitable for Radon transformation in three dimensions. One can use the correspondence between differential forms and vectors for translation. 


\section{B. The Radon transformation}

The Radon transform $\boldsymbol{F}_{\lambda}^{\mathcal{R}}(\boldsymbol{\kappa} \cdot \boldsymbol{x}, \boldsymbol{\kappa})$, (19) for $p=\boldsymbol{\kappa} \cdot \boldsymbol{x}$ which is the integral of the Trkalian field over the hyperplane orthogonal to $\boldsymbol{\kappa}$ containing $\boldsymbol{x}$ satisfies

$$
\nabla \times \boldsymbol{F}_{\lambda}^{\mathcal{R}}(\boldsymbol{\kappa} \cdot \boldsymbol{x}, \boldsymbol{\kappa})-\nu \boldsymbol{F}_{\lambda}^{\mathcal{R}}(\boldsymbol{\kappa} \cdot \boldsymbol{x}, \boldsymbol{\kappa})=0
$$

(11), where $\boldsymbol{\nabla} \cdot \boldsymbol{F}_{\lambda}^{\mathcal{R}}(\boldsymbol{\kappa} \cdot \boldsymbol{x}, \boldsymbol{\kappa})=0$.

We shall prove that the Radon transform of a Trkalian field satisfies a corresponding eigenvalue equation in the transform space. We shall interchangeably use [ $]^{\mathcal{R}}$ or $\mathcal{R}[$ ] for denoting the Radon transform. (See Appendix A 1].)

Definition 1: The operator $\boldsymbol{\Gamma}$ is defined as

$$
\boldsymbol{\Gamma}=\boldsymbol{\kappa} \frac{\partial}{\partial p}=\frac{\partial}{\partial p} \kappa
$$

Here $\partial / \partial p$ is called the (infinitesimal) parallel displacement operator of the plane $\stackrel{23}{ }^{23}$

Proposition 1: The Radon transform intertwines:

a) the curl operator $\boldsymbol{\nabla} \times$ and $\boldsymbol{\Gamma} \times$

$$
\mathcal{R}[\boldsymbol{\nabla} \times \boldsymbol{V}(\boldsymbol{x})](p, \boldsymbol{\kappa})=\boldsymbol{\Gamma} \times \mathcal{R}[\boldsymbol{V}(\boldsymbol{x})](p, \boldsymbol{\kappa}),
$$

b) the divergence operator $\boldsymbol{\nabla} \cdot$ and $\boldsymbol{\Gamma}$.

$$
\boldsymbol{\mathcal { R }}[\boldsymbol{\nabla} \cdot \boldsymbol{V}(\boldsymbol{x})](p, \boldsymbol{\kappa})=\boldsymbol{\Gamma} \cdot \boldsymbol{\mathcal { R }}[\boldsymbol{V}(\boldsymbol{x})](p, \boldsymbol{\kappa}),
$$

c) the gradient operator $\boldsymbol{\nabla}$ and $\boldsymbol{\Gamma}$

$$
\mathcal{R}[\boldsymbol{\nabla} f(\boldsymbol{x})](p, \boldsymbol{\kappa})=\boldsymbol{\Gamma} \mathcal{R}[f(\boldsymbol{x})](p, \boldsymbol{\kappa}) .
$$

Proof: We have

$$
\begin{aligned}
& \frac{\partial}{\partial x^{i}} \delta(p-\boldsymbol{\kappa} \cdot \boldsymbol{x})=-\kappa_{i} \delta^{\prime}(p-\boldsymbol{\kappa} \cdot \boldsymbol{x}), \quad \frac{\partial s}{\partial x^{i}}=-\kappa_{i}, \\
& \delta^{\prime}(p-\boldsymbol{\kappa} \cdot \boldsymbol{x})=\frac{d}{d s} \delta(s)=\frac{\partial}{\partial p} \delta(p-\boldsymbol{\kappa} \cdot \boldsymbol{x} \cdot \boldsymbol{x}), \quad \frac{\partial s}{\partial p}=1,
\end{aligned}
$$

which yield

$$
\begin{aligned}
\boldsymbol{\nabla} \delta(p-\boldsymbol{\kappa} \cdot \boldsymbol{x}) & =-\boldsymbol{\kappa} \delta^{\prime}(p-\boldsymbol{\kappa} \cdot \boldsymbol{x}) \\
& =-\boldsymbol{\kappa} \frac{\partial}{\partial p} \delta(p-\boldsymbol{\kappa} \cdot \boldsymbol{x})
\end{aligned}
$$

Thus 24,25 we find

$$
\begin{aligned}
\mathcal{R}\left[\frac{\partial}{\partial x^{i}} f(\boldsymbol{x})\right](p, \boldsymbol{\kappa}) & =\int_{D} \frac{\partial f(\boldsymbol{x})}{\partial x^{i}} \delta(p-\boldsymbol{\kappa} \cdot \boldsymbol{x}) d^{3} x \\
& =\kappa_{i} \frac{\partial}{\partial p} \boldsymbol{\mathcal { R }}[f(\boldsymbol{x})](p, \boldsymbol{\kappa}) .
\end{aligned}
$$

The domain $D$ of integration is the whole space: $D=\mathbb{R}^{3}$ unless otherwise stated. This leads to componentwise proofs of $\mathrm{a}, \mathrm{b}$ and $\mathrm{c}$. 
a) More precisely, consider a 3-dimesional (finite) region $D$ bounded by a surface $S=\partial D$. We have

$$
\begin{aligned}
\int_{D} \boldsymbol{\nabla} \times \boldsymbol{V}(\boldsymbol{x}) \delta(p-\boldsymbol{\kappa} \cdot \boldsymbol{x}) d^{3} x & =\int_{D} \boldsymbol{\nabla} \times[\boldsymbol{V}(\boldsymbol{x}) \delta(p-\boldsymbol{\kappa} \cdot \boldsymbol{x})] d^{3} x-\int_{D} \boldsymbol{\nabla} \delta(p-\boldsymbol{\kappa} \cdot \boldsymbol{x}) \times \boldsymbol{V}(\boldsymbol{x}) d^{3} x \\
& =-\int_{S} \delta(p-\boldsymbol{\kappa} \cdot \boldsymbol{x}) \boldsymbol{V}(\boldsymbol{x}) \times d \boldsymbol{s}-\int_{D} \boldsymbol{\nabla} \delta(p-\boldsymbol{\kappa} \cdot \boldsymbol{x}) \times \boldsymbol{V}(\boldsymbol{x}) d^{3} x
\end{aligned}
$$

The first integral vanishes if we consider functions $\boldsymbol{V}(\boldsymbol{x})$ which vanish at infinity, as the region $D$ is extended to whole space. (For a finite region we could use vectors normal to the surface.) Thus

$$
\boldsymbol{\nabla} \times \boldsymbol{V}(\boldsymbol{x}) \delta(p-\boldsymbol{\kappa} \cdot \boldsymbol{x})=-\boldsymbol{\nabla} \delta(p-\boldsymbol{\kappa} \cdot \boldsymbol{x}) \times \boldsymbol{V}(\boldsymbol{x}),
$$

(under integral). Hence we find

$$
\begin{aligned}
\boldsymbol{\mathcal { R }}[\boldsymbol{\nabla} \times \boldsymbol{V}(\boldsymbol{x})](p, \boldsymbol{\kappa}) & =\int_{D} \boldsymbol{\nabla} \times \boldsymbol{V}(\boldsymbol{x}) \delta(p-\boldsymbol{\kappa} \cdot \boldsymbol{x}) d^{3} x \\
& =\boldsymbol{\Gamma} \times \boldsymbol{\mathcal { R }}[\boldsymbol{V}(\boldsymbol{x})](p, \boldsymbol{\kappa})
\end{aligned}
$$

using (27) and (24).

The proofs of $\mathrm{b}$ and $\mathrm{c}$ are based on a similar reasoning. The $(\times)$ product is respectively replaced by $(\cdot)$ and ordinary product, with similar boundary conditions at infinity (for a finite region, with certain boundary conditions). See Ref. 11 for alternative proofs with different techniques.

Definition 2: The operator $\Gamma^{2}$ is defined as $\Gamma^{2}=\boldsymbol{\Gamma} \cdot \boldsymbol{\Gamma}$.

Proposition 2: The operator $\Gamma^{2}$ acts on $f^{\mathcal{R}}(p, \kappa)$ as

$$
\Gamma^{2} f^{\mathcal{R}}(p, \boldsymbol{\kappa})=\left(\partial^{2} / \partial p^{2}\right) f^{\mathcal{R}}(p, \boldsymbol{\kappa})
$$

Proof: We simply have

$$
\begin{aligned}
\Gamma^{2} f^{\mathcal{R}}(p, \boldsymbol{\kappa}) & =\boldsymbol{\Gamma} \cdot\left[\boldsymbol{\Gamma} f^{\mathcal{R}}(p, \boldsymbol{\kappa})\right] \\
& =\boldsymbol{\kappa} \frac{\partial}{\partial p} \cdot\left[\boldsymbol{\kappa} \frac{\partial}{\partial p} f^{\mathcal{R}}(p, \boldsymbol{\kappa})\right] \\
& =\frac{\partial}{\partial p}(\boldsymbol{\kappa} \cdot \boldsymbol{\kappa}) \frac{\partial}{\partial p} f^{\mathcal{R}}(p, \boldsymbol{\kappa}) \\
& =\frac{\partial^{2}}{\partial p^{2}} f^{\mathcal{R}}(p, \boldsymbol{\kappa}), \quad \boldsymbol{\kappa} \cdot \boldsymbol{\kappa}=1 .
\end{aligned}
$$

This operator corresponds to $\square=\Gamma^{2}$ which is intertwined 26 with Laplacian $\nabla^{2}$. This acts componentwise on vectors $\boldsymbol{V}^{R}(p, \boldsymbol{\kappa})$ analogous to the action of $\nabla^{2}$ on $\boldsymbol{V}(\boldsymbol{x})$.

Proposition 3: The operator $\boldsymbol{\Gamma}$ satisfies the following identities:

a) $\boldsymbol{\Gamma} \times\left[\boldsymbol{\Gamma} f^{\mathcal{R}}(p, \boldsymbol{\kappa})\right]=0$,

b) $\boldsymbol{\Gamma} \cdot\left[\boldsymbol{\Gamma} \times \boldsymbol{V}^{\mathcal{R}}(p, \boldsymbol{\kappa})\right]=0$,

c) $\boldsymbol{\Gamma} \times\left[\boldsymbol{\Gamma} \times \boldsymbol{V}^{\mathcal{R}}(p, \boldsymbol{\kappa})\right]=\boldsymbol{\Gamma}\left[\boldsymbol{\Gamma} \cdot \boldsymbol{V}^{\mathcal{R}}(p, \boldsymbol{\kappa})\right]-\Gamma^{2} \boldsymbol{V}^{\mathcal{R}}(p, \boldsymbol{\kappa})$.

Proof: These follow as

a)

$$
\begin{aligned}
\boldsymbol{\Gamma} \times\left[\boldsymbol{\Gamma} f^{\mathcal{R}}(p, \boldsymbol{\kappa})\right] & =\boldsymbol{\kappa} \frac{\partial}{\partial p} \times\left[\boldsymbol{\kappa} \frac{\partial}{\partial p} f^{\mathcal{R}}(p, \boldsymbol{\kappa})\right] \\
& =\frac{\partial}{\partial p}(\boldsymbol{\kappa} \times \boldsymbol{\kappa}) \frac{\partial}{\partial p} f^{\mathcal{R}}(p, \boldsymbol{\kappa}) \\
& =0, \quad \boldsymbol{\kappa} \times \boldsymbol{\kappa}=0
\end{aligned}
$$


b)

$$
\begin{aligned}
\boldsymbol{\Gamma} \cdot\left[\boldsymbol{\Gamma} \times \boldsymbol{V}^{\mathcal{R}}(p, \boldsymbol{\kappa})\right] & =\boldsymbol{\kappa} \frac{\partial}{\partial p} \cdot\left[\boldsymbol{\kappa} \frac{\partial}{\partial p} \times \boldsymbol{V}^{\mathcal{R}}(p, \boldsymbol{\kappa})\right] \\
& =\frac{\partial^{2}}{\partial p^{2}} \boldsymbol{\kappa} \cdot\left[\boldsymbol{\kappa} \times \boldsymbol{V}^{\mathcal{R}}(p, \boldsymbol{\kappa})\right] \\
& =0, \quad \boldsymbol{\kappa} \cdot\left[\boldsymbol{\kappa} \times \boldsymbol{V}^{\mathcal{R}}(p, \boldsymbol{\kappa})\right]=0,
\end{aligned}
$$

c)

$$
\begin{aligned}
\boldsymbol{\Gamma} \times\left[\boldsymbol{\Gamma} \times \boldsymbol{V}^{\mathcal{R}}(p, \boldsymbol{\kappa})\right] & =\boldsymbol{\kappa} \frac{\partial}{\partial p} \times\left[\boldsymbol{\kappa} \frac{\partial}{\partial p} \times \boldsymbol{V}^{\mathcal{R}}(p, \boldsymbol{\kappa})\right] \\
& =\frac{\partial^{2}}{\partial p^{2}} \boldsymbol{\kappa} \times\left[\boldsymbol{\kappa} \times \boldsymbol{V}^{\mathcal{R}}(p, \boldsymbol{\kappa})\right] \\
& =\frac{\partial^{2}}{\partial p^{2}}\left\{\left[\boldsymbol{\kappa} \cdot \boldsymbol{V}^{\mathcal{R}}(p, \boldsymbol{\kappa})\right] \boldsymbol{\kappa}-(\boldsymbol{\kappa} \cdot \boldsymbol{\kappa}) \boldsymbol{V}^{\mathcal{R}}(p, \boldsymbol{\kappa})\right\} \\
& =\boldsymbol{\kappa} \frac{\partial}{\partial p}\left[\boldsymbol{\kappa} \frac{\partial}{\partial p} \cdot \boldsymbol{V}^{\mathcal{R}}(p, \boldsymbol{\kappa})\right]-\boldsymbol{\kappa} \frac{\partial}{\partial p} \cdot\left[\boldsymbol{\kappa} \frac{\partial}{\partial p} \boldsymbol{V}^{\mathcal{R}}(p, \boldsymbol{\kappa})\right] \\
& =\boldsymbol{\Gamma}\left[\boldsymbol{\Gamma} \cdot \boldsymbol{V}^{\mathcal{R}}(p, \boldsymbol{\kappa})\right]-\Gamma^{2} \boldsymbol{V}^{\mathcal{R}}(p, \boldsymbol{\kappa}) .
\end{aligned}
$$

Proposition 4: The Radon transform intertwines the eigenvalue operators $(\nabla \times)-\nu=0$ and $(\boldsymbol{\Gamma} \times)-\nu=0$ for constant eigenvalues.

Proof: The proof follows from the linearity of the Radon transformation.

We can easily check the Radon transform (19) satisfies

$$
\boldsymbol{\Gamma} \times \boldsymbol{F}_{\lambda}^{\mathcal{R}}(p, \boldsymbol{\kappa})-\nu \boldsymbol{F}_{\lambda}^{\mathcal{R}}(p, \boldsymbol{\kappa})=0
$$

[in differential forms: $d p=\kappa=-\omega^{3}$, (4) since $p=\boldsymbol{\kappa} \cdot \boldsymbol{x}$ ]. We also have: $\boldsymbol{\kappa} \cdot \boldsymbol{F}_{\lambda}^{\mathcal{R}}(p, \boldsymbol{\kappa})=0$ which leads to $\boldsymbol{\Gamma} \cdot \boldsymbol{F}_{\lambda}^{\mathcal{R}}(p, \boldsymbol{\kappa})=0$. We can write (29) as

$$
\frac{\partial}{\partial p} \boldsymbol{F}_{\lambda}^{\mathcal{R}}(p, \boldsymbol{\kappa})+\nu \kappa \times \boldsymbol{F}_{\lambda}^{\mathcal{R}}(p, \boldsymbol{\kappa})=0
$$

A change of sign: $p \longrightarrow-p$ or $\kappa \longrightarrow-\kappa$ (but not both) leads to a change of sign in the eigen-value: $\nu \longrightarrow-\nu$ in (29). We shall discuss this again in Section $\nabla$. The Radon transform $\boldsymbol{\Gamma} U^{\mathcal{R}}(p, \boldsymbol{\kappa})$ of an arbitrary gauge transformation $\boldsymbol{\nabla} U(\boldsymbol{x})$ is normal $\underline{\underline{6}}$ to $\mathbb{S}^{2}$.

We need the adjoint Radon transformation for discussion in the reverse direction.

Definition 3: The adjoint ${ }^{26,27}$ Radon transform of a function $\boldsymbol{G}(p, \boldsymbol{\kappa})$ on the transform space is

$$
\boldsymbol{G}^{\mathcal{R}^{\dagger}}(\boldsymbol{x})=\mathcal{R}^{\dagger}[\boldsymbol{G}(p, \boldsymbol{\kappa})](\boldsymbol{x})=\int_{S^{2}} \boldsymbol{G}(\boldsymbol{\kappa} \cdot \boldsymbol{x}, \boldsymbol{\kappa}) d \Omega .
$$

The function $\boldsymbol{G}(\boldsymbol{\kappa} \cdot \boldsymbol{x}, \boldsymbol{\kappa})$ is to be taken as $\boldsymbol{G}(\boldsymbol{\kappa} \cdot \boldsymbol{x}, \boldsymbol{\kappa})=\boldsymbol{F}^{\mathcal{R}}(\boldsymbol{\kappa} \cdot \boldsymbol{x}, \boldsymbol{\kappa})$. The Radon transform $\mathcal{R}$ integrates over the set of points in a hyperplane, the adjoint transform $\mathcal{R}^{\dagger}$ integrates over the set of hyperplanes through a point. 26,28

Proposition 5: The adjoint Radon transform intertwines:

a) the operators $\boldsymbol{\Gamma} \times$ and curl $\boldsymbol{\nabla} \times$

$$
\mathcal{R}^{\dagger}[\boldsymbol{\Gamma} \times \boldsymbol{G}(p, \boldsymbol{\kappa})](\boldsymbol{x})=\boldsymbol{\nabla} \times \mathcal{R}^{\dagger}[\boldsymbol{G}(p, \boldsymbol{\kappa})](\boldsymbol{x}),
$$

b) the operators $\boldsymbol{\Gamma} \cdot$ and divergence $\boldsymbol{\nabla}$.

$$
\mathcal{R}^{\dagger}[\boldsymbol{\Gamma} \cdot \boldsymbol{G}(p, \boldsymbol{\kappa})](\boldsymbol{x})=\boldsymbol{\nabla} \cdot \boldsymbol{\mathcal { R }}^{\dagger}[\boldsymbol{G}(p, \boldsymbol{\kappa})](\boldsymbol{x}),
$$

c) the operators $\boldsymbol{\Gamma}$ and gradient $\boldsymbol{\nabla}$

$$
\mathcal{R}^{\dagger}[\boldsymbol{\Gamma} \phi(p, \boldsymbol{\kappa})](\boldsymbol{x})=\boldsymbol{\nabla} \boldsymbol{\mathcal { R }}^{\dagger}[\phi(p, \boldsymbol{\kappa})](\boldsymbol{x}) .
$$


Proof: The componentwise proofs of $\mathrm{a}, \mathrm{b}$ and $\mathrm{c}$ follow from the identity

$$
\begin{aligned}
\frac{\partial}{\partial x^{i}} \mathcal{R}^{\dagger}[\phi(p, \boldsymbol{\kappa})](\boldsymbol{x}) & =\frac{\partial}{\partial x^{i}} \int_{S^{2}} \phi(\boldsymbol{\kappa} \cdot \boldsymbol{x}, \boldsymbol{\kappa}) d \Omega \\
& =\int_{S^{2}} \kappa^{i} \frac{\partial}{\partial p} \phi(p, \boldsymbol{\kappa}) d \Omega, \quad p=\boldsymbol{\kappa} \cdot \boldsymbol{x} \\
& =\mathcal{R}^{\dagger}\left[\kappa^{i} \frac{\partial}{\partial p} \phi(p, \boldsymbol{\kappa})\right](\boldsymbol{x}) .
\end{aligned}
$$

The adjoint Radon transform also intertwines ${ }^{26}$ the operators $\Gamma^{2}$ and $\nabla^{2}$.

Proposition 6: The adjoint Radon transform intertwines the eigenvalue operators $(\boldsymbol{\Gamma} \times)-\nu=0$ and $(\boldsymbol{\nabla} \times)-\nu=0$ for constant eigenvalues.

Proof: The proof follows from the linearity of the adjoint Radon transformation.

Thus, we have a correspondence between the (constant) eigenvalue equations $(\boldsymbol{\nabla} \times)-\nu=0$ in the physical space and $(\boldsymbol{\Gamma} \times)-\nu=0$ in the transform space. Note that we should employ $\left(-1 / 8 \pi^{2}\right) \nabla^{2}$ on $\boldsymbol{\mathcal { R }}^{\dagger}\left[\boldsymbol{F}^{\mathcal{R}}(p, \boldsymbol{\kappa})\right]$ for a complete inversion. This simplifies for a Trkalian field. Further one should consider the appropriate class of functions for the Radon transform and its adjoint $\underline{\underline{26}}$ (See Appendix A 1)

\section{A refinement}

The Radon transform (19) of a Trkalian field is composed of both helicity components of the field. The inverse Radon transform (A4) requires knowledge of the transform evaluated over the entire sphere $\stackrel{16}{\underline{16}}$ However the knowledge of a helicity component evaluated over the entire sphere suffices to reproduce the knowledge of the other component. Thus construction of the field can be achieved using the knowledge of a single component evaluated over the entire sphere as in (16). Equivalently, the field can be constructed using knowledge of both components, i. e. the Radon transform (19), on a canonical hemisphere which can consist of disconnected parts. For scalar fields, a similar result is proved in Ref. 16. Here, this easily follows as one considers both helicity components in the Radon transform of a Trkalian field. This also removes redundancy of double-covering of space. $\underline{\underline{16}}$

A canonical hemisphere $H$ is a (Lebesgue measurable) subset of the unit 2-sphere $\mathbb{S}^{2}$ whose area is $2 \pi$ such that if the tip of a unit vector $\boldsymbol{\kappa}$ based at the origin is in the hemisphere then the tip of $-\boldsymbol{\kappa}$ is not in the hemispere 16 The complementary set $H^{\prime}$ in $\mathbb{S}^{2}$ is a canonical hemisphere too. A canonical hemisphere can consists of disconnected parts.

If we substitute (19) with $p=\boldsymbol{\kappa} \cdot \boldsymbol{x}$ in (A4), we find

$$
\begin{aligned}
\boldsymbol{F}_{\lambda}(\boldsymbol{x}) & =-\frac{1}{(2 \pi)^{3 / 2}} \frac{1}{g} \frac{1}{\nu^{2}} \nabla_{x}^{2} \int_{H}\left[e^{i \lambda \nu \boldsymbol{\kappa} \cdot \boldsymbol{x}} \boldsymbol{Q}_{\lambda}(\boldsymbol{\kappa}) s_{\lambda}(\lambda \nu \boldsymbol{\kappa})+e^{-i \lambda \nu \boldsymbol{\kappa} \cdot \boldsymbol{x}} \boldsymbol{Q}_{\lambda}(-\boldsymbol{\kappa}) s_{\lambda}(-\lambda \nu \boldsymbol{\kappa})\right] d \Omega \\
& =\frac{1}{(2 \pi)^{3 / 2}} \frac{1}{g} \int_{H}\left[e^{i \lambda \nu \boldsymbol{\kappa} \cdot \boldsymbol{x}} \boldsymbol{Q}_{\lambda}(\boldsymbol{\kappa}) s_{a}(\lambda \nu \boldsymbol{\kappa})+e^{-i \lambda \nu \boldsymbol{\kappa} \cdot \boldsymbol{x}} \boldsymbol{Q} \lambda(-\boldsymbol{\kappa}) s_{\lambda}(-\lambda \nu \boldsymbol{\kappa})\right] d \Omega,
\end{aligned}
$$

as $\kappa \longrightarrow-\boldsymbol{\kappa}$ in $H^{\prime}$, using the identities (21). This can also be inferred from (16).

We can see this on a simple example choosing $s_{\lambda}(\lambda \nu \boldsymbol{\kappa})=(2 \pi)^{3 / 2} g \delta\left(\boldsymbol{\kappa}-\boldsymbol{\kappa}_{0}\right)$. We decompose the sphere $\mathbb{S}^{2}$ into two complementary canonical hemispheres $H$ and $H^{\prime}$ where the tip of $\boldsymbol{\kappa}_{0}$ is contained in $H: \boldsymbol{\kappa}_{0} \in H$. If we take $s_{\lambda}(-\lambda \nu \boldsymbol{\kappa})=(2 \pi)^{3 / 2} g \delta\left(\boldsymbol{\kappa}-\boldsymbol{\kappa}_{0}^{\prime}\right),\left(\boldsymbol{\kappa} \longrightarrow-\boldsymbol{\kappa}: \boldsymbol{\kappa}_{0} \longrightarrow \boldsymbol{\kappa}_{0}^{\prime}=-\boldsymbol{\kappa}_{0}\right)$ then $\boldsymbol{\kappa}_{0}^{\prime} \in H^{\prime}$. We find

$$
\begin{aligned}
\boldsymbol{F}_{\lambda}(\boldsymbol{x}) & =e^{i \lambda \nu \boldsymbol{\kappa}_{0} \cdot \boldsymbol{x}} \boldsymbol{Q}_{\lambda}\left(\boldsymbol{\kappa}_{0}\right) \\
& =e^{-i \lambda \nu \boldsymbol{\kappa}_{0}^{\prime} \cdot \boldsymbol{x}} \boldsymbol{Q}_{\lambda}\left(-\boldsymbol{\kappa}_{0}^{\prime}\right),
\end{aligned}
$$

using (32) or equivalently inverting it and integrating over $H^{\prime}$.

It is possible to define a left and right inverse of this refined (inverse) transformation if it is applied to Radon transforms of functions (see Appendix A2). Alternatively, one can introduce inverse of this refined transformation redefining the Radon transform and the set $\left\{\boldsymbol{F}^{\mathcal{R}}(p, \boldsymbol{\kappa})\right\}$ to require that $\boldsymbol{\kappa}$ be restricted into a canonical hemisphere. Then $\boldsymbol{\mathcal { R }}_{H}^{-1}$ is both a left and right inverse of $\boldsymbol{\mathcal { R }}_{H}: \boldsymbol{\mathcal { R }}_{H}^{-1} \boldsymbol{\mathcal { R }}_{H}[\boldsymbol{F}](\boldsymbol{x})=\boldsymbol{F}(\boldsymbol{x}), \boldsymbol{\mathcal { R }}_{H} \boldsymbol{\mathcal { R }}_{H}^{-1}\left[\boldsymbol{F}^{\mathcal{R}}\right](p, \boldsymbol{\kappa})=\boldsymbol{F}^{\mathcal{R}}(p, \boldsymbol{\kappa}) \underline{\underline{16}}$ 


\section{RADON TRANSFORM AND BIOT-SAVART INTEGRAL}

In this section, we shall consider the relation of the Radon transformation with Riesz potential and $B S$ integral. We shall also present an Ampere law type relation for Trkalian fields. Then we shall discuss Radon transform of the $B S$ integral.

We can easily ${ }^{25-28}$ show

$$
\boldsymbol{\mathcal { R }}^{\dagger} \boldsymbol{\mathcal { R }}[\boldsymbol{F}](\boldsymbol{x})=8 \pi^{2} I^{2}[\boldsymbol{F}](\boldsymbol{x})
$$

The integral on the right-hand side which is defined as the convolution of $\boldsymbol{F}$ with Riesz kernel $K_{2}=(1 / 4 \pi)|\boldsymbol{x}|^{-1}$

$$
\begin{aligned}
I^{2}[\boldsymbol{F}](\boldsymbol{x}) & =\left(K_{2} * \boldsymbol{F}\right)(\boldsymbol{x}) \\
& =\frac{1}{4 \pi} \int_{D} \frac{\boldsymbol{F}(\boldsymbol{y})}{|\boldsymbol{x}-\boldsymbol{y}|} d^{3} y
\end{aligned}
$$

is called the Riesz potential for the vector field $\boldsymbol{F}(\boldsymbol{x})$. We remind: $D=\mathbb{R}^{3}$ for the Radon transformation. The operator $-\nabla^{2}$ is an (left) inverse of the Riesz integral. 28

We find

$$
\boldsymbol{\nabla}_{x} \times \mathcal{R}^{\dagger} \boldsymbol{\mathcal { R }}[\boldsymbol{F}](\boldsymbol{x})=8 \pi^{2} B S[\boldsymbol{F}](\boldsymbol{x})
$$

where

$$
\begin{aligned}
B S[\boldsymbol{F}](\boldsymbol{x}) & =\boldsymbol{\nabla}_{x} \times I^{2}[\boldsymbol{F}](\boldsymbol{x}) \\
& =\frac{1}{4 \pi} \int_{D} \boldsymbol{F}(\boldsymbol{y}) \times \frac{\boldsymbol{x}-\boldsymbol{y}}{|\boldsymbol{x}-\boldsymbol{y}|^{3}} d^{3} y
\end{aligned}
$$

is the $B S$ integral operator ${ }^{17}$ The vector potential for $B S[\boldsymbol{F}](\boldsymbol{x})$ is given by the Riesz potential (35).17

We immediately see that

$$
\nabla_{x} \cdot \nabla_{x} \times \mathcal{R}^{\dagger} \mathcal{R}[\boldsymbol{F}](\boldsymbol{x})=8 \pi^{2} \boldsymbol{\nabla}_{x} \cdot B S[\boldsymbol{F}](\boldsymbol{x})=0
$$

We also find

$$
\boldsymbol{\nabla}_{x} \times \boldsymbol{\nabla}_{x} \times \mathcal{R}^{\dagger} \boldsymbol{\mathcal { R }}[\boldsymbol{F}](\boldsymbol{x})=8 \pi^{2} \boldsymbol{\nabla}_{x} \times B S[\boldsymbol{F}](\boldsymbol{x})=8 \pi^{2} \boldsymbol{F}(\boldsymbol{x})
$$

if $\boldsymbol{F}$ is divergence-free and it also vanishes at infinity (for a finite region $D$, it is tangent to the surface $S=\partial D$ bounding this region). (See Appendix C.) Hence the $B S$ integral is divergence-free and the curl operator is a left inverse of this under these conditions, 17,29

We can associate an eigenvalue equation for $B S$ operator (the eigenvalue being reciprocal of $\nu$ ) with the Trkalian

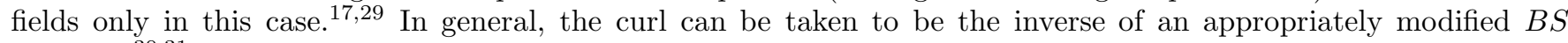
operator 30,31

As for the Trkalian fields, we find

$$
\boldsymbol{\mathcal { R }}^{\dagger} \boldsymbol{\mathcal { R }}\left[\boldsymbol{F}_{\lambda}\right](\boldsymbol{x})=8 \pi^{2} \frac{1}{\nu^{2}} \boldsymbol{F}_{\lambda}(\boldsymbol{x})
$$

using Definition 3 and (19), (16). This can be inferred from (A4) using $\Gamma^{2}$ which is intertwined with $\nabla^{2}$. Note that the conditions in (39) are satisfied for a Trkalian field if its Radon transform exists (see Appendix A 1 ). The equation (40) yields

$$
\boldsymbol{\nabla}_{x} \times \boldsymbol{\nabla}_{x} \times \boldsymbol{\mathcal { R }}^{\dagger} \boldsymbol{\mathcal { R }}\left[\boldsymbol{F}_{\lambda}\right](\boldsymbol{x})=8 \pi^{2} \boldsymbol{F}_{\lambda}(\boldsymbol{x})
$$


This reduces to the inverse Radon transform (A4) using (22) on the left-hand side.

We find an Ampere law type relation ${ }^{4.5}$

$$
\Phi=\nu Q,
$$

integrating the equation (11) over a surface $S$ bounded by the curve $C=\partial S$. Here

$$
Q=\int_{S} \boldsymbol{F}(\boldsymbol{x}) \cdot d \boldsymbol{s}, \quad \Phi=\int_{S}[\boldsymbol{\nabla} \times \boldsymbol{F}(\boldsymbol{x})] \cdot d \boldsymbol{s},
$$

are respectively the flux of $\boldsymbol{F}(\boldsymbol{x})$ and $\boldsymbol{\nabla} \times \boldsymbol{F}(\boldsymbol{x})$ through the surface $S$. The flux $\Phi$ reduces to

$$
\Phi=\int_{C} \boldsymbol{F}(\boldsymbol{x}) \cdot d \boldsymbol{l}
$$

the circulation of $\boldsymbol{F}(\boldsymbol{x})$ on $C$. Thus the flux of a Trkalian field through a surface is determined by its circulation on the boundary of the surface $\frac{4,5}{, 5}$ Note that the equation (22) yields an analogous relation for $\boldsymbol{F}_{\lambda}^{\mathcal{R}}(\boldsymbol{\kappa} \cdot \boldsymbol{x}, \boldsymbol{\kappa})$.

\section{A. Radon transform of the Biot-Savart integral}

We shall make use of Fourier slice theorem 26,27

$$
\mathcal{F}\left[\boldsymbol{F}^{\mathcal{R}}(p, \boldsymbol{\kappa})\right](k, \boldsymbol{\kappa})=2 \pi \mathcal{F}[\boldsymbol{F}(\boldsymbol{x})](k \boldsymbol{\kappa}),
$$

for finding Radon transform of the $B S$ integral. On the left-hand side $\mathcal{F}$ stands for 1-dimensional Fourier transform in the first variable whereas on the right-hand side it is a vector Fourier transform in 3 dimensions. We also need Fourier transform of the Riesz $\frac{26,27}{2 n}$ integral

$$
\begin{aligned}
\mathcal{F}\left\{I^{2}[\boldsymbol{F}](\boldsymbol{x})\right\}(\boldsymbol{k}) & =\frac{1}{k^{2}} \mathcal{F}[\boldsymbol{F}(\boldsymbol{x})](k \boldsymbol{\kappa}) \\
& =\frac{1}{2 \pi} \frac{1}{k^{2}} \mathcal{F}\left[\boldsymbol{F}^{\mathcal{R}}(q, \boldsymbol{\kappa})\right](k, \boldsymbol{\kappa}) .
\end{aligned}
$$

Here the last line follows from (45).

We find

$$
\begin{aligned}
\mathcal{F}\left\{I^{2}[\boldsymbol{F}]^{\mathcal{R}}(p, \boldsymbol{\kappa})\right\}(k, \boldsymbol{\kappa}) & =2 \pi \mathcal{F}\left\{I^{2}[\boldsymbol{F}](\boldsymbol{x})\right\}(k \boldsymbol{\kappa}) \\
& =\frac{1}{k^{2}} \mathcal{F}\left[\boldsymbol{F}^{\mathcal{R}}(q, \boldsymbol{\kappa})\right](k, \boldsymbol{\kappa}),
\end{aligned}
$$

using (45) and (46). This yields Radon transform of the Riesz integral

$$
\begin{aligned}
\mathcal{R}\left\{I^{2}[\boldsymbol{F}](\boldsymbol{x})\right\}(p, \boldsymbol{\kappa}) & =\mathcal{F}^{-1}\left\{\frac{1}{k^{2}} \mathcal{F}\left[\boldsymbol{F}^{\mathcal{R}}(q, \boldsymbol{\kappa})\right](k, \boldsymbol{\kappa})\right\}(p, \boldsymbol{\kappa}) \\
& =\frac{1}{2 \pi} \int\left[\int e^{i k(p-q)} \frac{1}{k^{2}} d k\right] \boldsymbol{F}^{R}(q, \boldsymbol{\kappa}) d q .
\end{aligned}
$$

This equivalently corresponds to $\mathcal{R}^{\dagger} \boldsymbol{\mathcal { R }}[\boldsymbol{F}](\boldsymbol{x})$, (34) $\underline{32}$ This is a convolution for the Radon transformation. $\frac{26,27}{2}$ The operator $-\Gamma^{2}$ is an (left) inverse of the Radon transform of the Riesz integral. We define Radon-Biot-Savart integral operator as

$$
\begin{aligned}
R B S\left[\boldsymbol{F}^{\mathcal{R}}(q, \boldsymbol{\kappa})\right](p, \boldsymbol{\kappa}) & =B S[\boldsymbol{F}]^{\mathcal{R}}(p, \boldsymbol{\kappa}) \\
& =\boldsymbol{\Gamma}_{p} \times \mathcal{F}^{-1}\left\{\frac{1}{k^{2}} \mathcal{F}\left[\boldsymbol{F}^{\mathcal{R}}(q, \boldsymbol{\kappa})\right](k, \boldsymbol{\kappa})\right\}(p, \boldsymbol{\kappa}) \\
& =\mathcal{F}^{-1}\left\{\frac{1}{k^{2}} \mathcal{F}\left[\boldsymbol{\Gamma}_{q} \times \boldsymbol{F}^{\mathcal{R}}(q, \boldsymbol{\kappa})\right](k, \boldsymbol{\kappa})\right\}(p, \boldsymbol{\kappa}),
\end{aligned}
$$


the Radon transform of the $B S$ integral using Proposition 1 and (37), (48).

We immediately see that

$$
\boldsymbol{\Gamma} \cdot R B S\left[\boldsymbol{F}^{\mathcal{R}}(q, \boldsymbol{\kappa})\right](p, \boldsymbol{\kappa})=0
$$

using Proposition 3 . We find

$$
\begin{aligned}
\boldsymbol{\Gamma} \times R B S\left[\boldsymbol{F}^{\mathcal{R}}(q, \boldsymbol{\kappa})\right](p, \boldsymbol{\kappa}) & =-\boldsymbol{\kappa} \times\left[\boldsymbol{\kappa} \times \boldsymbol{F}^{\mathcal{R}}(p, \boldsymbol{\kappa})\right] \\
& =-\left\{\left[\boldsymbol{\kappa} \cdot \boldsymbol{F}^{\mathcal{R}}(p, \boldsymbol{\kappa})\right] \boldsymbol{\kappa}-(\boldsymbol{\kappa} \cdot \boldsymbol{\kappa}) \boldsymbol{F}^{\mathcal{R}}(p, \boldsymbol{\kappa})\right\}
\end{aligned}
$$

This yields

$$
\boldsymbol{\Gamma} \times R B S\left[\boldsymbol{F}^{\mathcal{R}}(q, \boldsymbol{\kappa})\right](p, \boldsymbol{\kappa})=\boldsymbol{F}^{\mathcal{R}}(p, \boldsymbol{\kappa}),
$$

if $\boldsymbol{\kappa} \cdot \boldsymbol{F}^{\mathcal{R}}=0$. This condition leads to $\boldsymbol{\Gamma} \cdot \boldsymbol{F}^{\mathcal{R}}=0$ [as can be inferred from [49)] which corresponds to $\boldsymbol{\nabla} \cdot \boldsymbol{F}=0$ in the physical space. Thus we conclude that $\boldsymbol{\Gamma} \times$ is a left inverse of the $R B S$ operator, if $\boldsymbol{\kappa} \cdot \boldsymbol{F}^{\mathcal{R}}=0$. We can associate an eigenvalue equation for $R B S$ operator with the Radon transform of Trkalian fields.

The $R B S$ operator also acts as a left inverse

$$
R B S\left[\boldsymbol{\Gamma}_{q} \times \boldsymbol{F}^{\mathcal{R}}(q, \boldsymbol{\kappa})\right](p, \boldsymbol{\kappa})=\boldsymbol{F}^{\mathcal{R}}(p, \boldsymbol{\kappa}),
$$

of the operator $\boldsymbol{\Gamma} \times$ for those vector fields $\boldsymbol{F}^{\mathcal{R}}$ with $\boldsymbol{\Gamma} \cdot \boldsymbol{F}^{\mathcal{R}}=0$. For example, the Radon transform (19) of a Trkalian field is an eigenvector

$$
R B S\left[\boldsymbol{F}_{\lambda}^{\mathcal{R}}(q, \boldsymbol{\kappa})\right](p, \boldsymbol{\kappa})=\frac{1}{\nu} \boldsymbol{F}_{\lambda}^{\mathcal{R}}(p, \boldsymbol{\kappa}),
$$

of the $R B S$ operator with reciprocal eigenvalue. The kernel of the $R B S$ operator consists of those vector fields for which $\boldsymbol{\Gamma} \times \boldsymbol{F}^{\mathcal{R}}=0$ i. e. gauge terms $\boldsymbol{\Gamma} U^{\mathcal{R}}$, (Proposition 3) corresponding to gradient fields $\boldsymbol{\nabla} U$ in the physical space $\stackrel{17}{=}$

\section{EXAMPLES}

In this section we shall first present applications of our constructions on the $\mathrm{L}$ solution. Then we shall briefly discuss the CK method with circular and elliptic cylindrical solutions. We shall make use of an analogous method for finding solution of the corresponding equation in the transform space.

\section{A. Lundquist solution}

The $\mathrm{L}^{18}$ solution is

$$
\boldsymbol{F}_{L}=F_{0}\left[J_{1}(\nu r) \boldsymbol{e}_{\theta}+J_{0}(\nu r) \boldsymbol{e}_{z}\right],
$$

where $J_{m}$ is the Bessel function of order $m$ and $F_{0}$ is the strength of the field on $z$-axis. Note this is of helicity $\lambda=1, \underline{10}$ The spherical curl transform (17) of (55) is given by

$$
s_{1}(\nu \boldsymbol{\kappa})=-2^{1 / 2}(2 \pi)^{1 / 2} g F_{0} \delta\left(\kappa_{z}\right) e^{-i \psi} .
$$

The radial degree of freedom $k$ is also taken into account $\underline{10}$ Here: $\boldsymbol{k}=k_{r} \cos \psi \boldsymbol{e}_{x}+k_{r} \sin \psi \boldsymbol{e}_{y}+k_{z} \boldsymbol{e}_{z}$ in the circular cylindrical coordinates in transform space. Thus the spherical curl transform of the L field lives on the equatorial circle of the sphere $\underline{\underline{10}}$ 
This solution provides a simple example of Ampere law. Consider a disc $S$ which is bounded by the circle $C=\partial S$ of radius $R$, centered at the origin in $x y$-plane. We find

$$
\Phi=\nu Q=2 \pi F_{0} R J_{1}(\nu R)
$$

(42). Note that $\Phi=Q=0$ at zeros of $J_{1}(\nu R)$.

The $\mathrm{L}$ field is an eigenvector of the $B S$ operator

$$
B S\left[\boldsymbol{F}_{L}\right](\boldsymbol{x})=\frac{1}{\nu} \boldsymbol{F}_{L}(\boldsymbol{x}),
$$

(37). This calculation is given in Appendix D

We find

$$
\boldsymbol{F}_{L}^{\mathcal{R}}(p, \boldsymbol{\kappa})=2 \pi i F_{0} \frac{1}{\nu^{2}} \delta\left(\kappa_{z}\right)\left(e^{i \nu p} \boldsymbol{L}+e^{-i \nu p} \boldsymbol{L}^{\prime}\right)
$$

where $\boldsymbol{L}=\sin \psi \boldsymbol{e}_{x}-\cos \psi \boldsymbol{e}_{y}-i \boldsymbol{e}_{z}$ and $\boldsymbol{L}^{\prime}=-\sin \psi \boldsymbol{e}_{x}+\cos \psi \boldsymbol{e}_{y}-i \boldsymbol{e}_{z}$. Note that we have taken the cases: $k$ negative or positive into account here. We immediately see that $\boldsymbol{F}_{L}^{\mathcal{R}}(p, \boldsymbol{\kappa})$ satisfies the equation (29) anticipating the effect of $\delta\left(\kappa_{z}\right){ }^{10}$ This is also an eigenvector (54) of the $R B S$ operator.

\section{B. Chandrasekhar-Kendall cylindrical solutions}

Chandrasekhar-Kendall ${ }^{19}$ developed a method for deriving solutions to $\boldsymbol{\nabla} \times \boldsymbol{F}=\nu \boldsymbol{F}$ from the scalar Helmholtz equation. See also Refs. 33 35.

We find

$$
\left(\nabla^{2}+\nu^{2}\right) \boldsymbol{F}=0
$$

applying curl on $\boldsymbol{\nabla} \times \boldsymbol{F}-\nu \boldsymbol{F}=0$. Thus every solution of $\boldsymbol{\nabla} \times \boldsymbol{F}-\nu \boldsymbol{F}=0$ is a solution of (60), although the converse is not necessarily true. If $\Psi$ is a scalar function satisfying the Helmholtz equation

$$
\left(\nabla^{2}+\nu^{2}\right) \Psi=0
$$

then three independent solutions $\frac{19}{9}$ of the equation (60) are

$$
\boldsymbol{p}=\boldsymbol{\nabla} \times(\Psi \omega), \quad \boldsymbol{q}=\frac{1}{\nu} \nabla \times \boldsymbol{p}=\frac{1}{\nu} \nabla \times \nabla \times(\Psi \omega), \quad \boldsymbol{r}=\nabla \Psi
$$

Here $\boldsymbol{\omega}$ is a fixed vector (of unit norm). The vectors of type $\boldsymbol{p}$ and $\boldsymbol{q}$ are respectively called toroidal and poloidal. ${ }^{36-38}$ We also have: $\boldsymbol{\nabla} \times \boldsymbol{q}=\nu \boldsymbol{p}$. Hence we find: $\boldsymbol{\nabla} \times(\boldsymbol{p}+\boldsymbol{q})=\nu(\boldsymbol{p}+\boldsymbol{q})$. Therefore, the most general ${ }^{7,19}$ solution of $\boldsymbol{\nabla} \times \boldsymbol{F}-\nu \boldsymbol{F}=0$ among the solutions of (60) is

$$
\boldsymbol{F}=\boldsymbol{p}+\boldsymbol{q}=\boldsymbol{\nabla} \times(\Psi \boldsymbol{\omega})+\frac{1}{\nu} \nabla \times \nabla \times(\Psi \boldsymbol{\omega})
$$

The scalar $\Psi$ is called Debye potential. 39 This method has been frequently used for discussing circular cylindrical, spherical and other type solutions ${ }^{7}$ For the relation with Wu-Yang type solutions in the topologically massive Abelian gauge theory see Refs. 4,5 .

The $\mathrm{CK}^{19}$ solution in cylindrical coordinates is given by

$$
\boldsymbol{F}=-\left[\sigma \nabla \times\left(\Psi \boldsymbol{e}_{z}\right)+\nabla \times \nabla \times\left(\Psi \boldsymbol{e}_{z}\right)\right]
$$

where $\boldsymbol{\nabla} \times \boldsymbol{F}-\sigma \boldsymbol{F}=0$. In the circular ${ }^{40,41}$ cylindrical coordinates 


$$
\Psi(r, \theta, z)=J_{m}(\nu r) e^{i m \theta-i k z}, \quad \sigma^{2}=\nu^{2}+k^{2} .
$$

This reduces to the $\mathrm{L}$ solution (55) for $m=0, k=0,\left(F_{0}=-\nu^{2}\right)$. In the elliptic cylindrical coordinates the solution is given by (64) with

$$
\Psi(u, v, z)=U(u) V(v) e^{-i k z}, \quad \sigma^{2}=k^{2}+m^{2}=\nu^{2} .
$$

Here $U(u)$ and $V(v)$ are respectively the modified Mathieu function and the Mathieu function. For $k=0, \sigma=m=\nu$ and this solution reduces to Vandas-Romashets ${ }^{42.43}(\mathrm{VR})$ solution which is an elliptic generalization of L.

\section{Chandrasekhar-Kendall method in transform space}

We can adapt the CK method in the transform space for finding solutions to $\boldsymbol{\Gamma} \times \boldsymbol{G}=\nu \boldsymbol{G}$. Briefly, the solution of

$$
\boldsymbol{\Gamma} \times \boldsymbol{G}(p, \boldsymbol{\kappa})-\nu \boldsymbol{G}(p, \boldsymbol{\kappa})=0,
$$

that is to be found among the solutions of

$$
\left(\Gamma^{2}+\nu^{2}\right) \boldsymbol{G}(p, \boldsymbol{\kappa})=0,
$$

is

$$
\boldsymbol{G}(p, \boldsymbol{\kappa})=\boldsymbol{\Gamma} \times(\Psi \boldsymbol{\omega})+\frac{1}{\nu} \boldsymbol{\Gamma} \times \boldsymbol{\Gamma} \times(\Psi \boldsymbol{\omega}) .
$$

Here $\Psi(p, \boldsymbol{\kappa})$ satisfy

$$
\left(\Gamma^{2}+\nu^{2}\right) \Psi=0,
$$

and $\boldsymbol{\omega}$ is a fixed vector in the transform space. We have ignored the superscript $R$ for Radon transform in $\Psi \boldsymbol{\omega}$. We remark a few important issues. First, the Radon transform is defined on $\mathbb{S}^{2} \times \mathbb{R}$ [or $\left(\mathbb{R}^{3}-\{0\}\right) \times \mathbb{R}$ ], (see appendix A 1). Secondly, $\boldsymbol{G}(p, \boldsymbol{\kappa})$ may consist of distributions. The third issue is imposing boundary conditions in the transform space.

The first term in (69): $\boldsymbol{G}(p, \boldsymbol{\kappa})=\boldsymbol{\Gamma} \times(\Psi \boldsymbol{\omega})$ with $\Psi=\left[(2 \pi)^{2} / \nu^{3}\right]\left[e^{i \lambda \nu p} \delta\left(\boldsymbol{\kappa}-\boldsymbol{\kappa}_{0}\right)+e^{-i \lambda \nu p} \delta\left(\boldsymbol{\kappa}+\boldsymbol{\kappa}_{0}\right)\right]$ and $\boldsymbol{\omega}=\boldsymbol{Q}_{\lambda}\left(\boldsymbol{\kappa}_{0}\right)$ immediately yields

$$
\boldsymbol{G}(p, \boldsymbol{\kappa})=(2 \pi)^{2} \frac{1}{\nu^{2}}\left[e^{i \lambda \nu p} \delta\left(\boldsymbol{\kappa}-\boldsymbol{\kappa}_{0}\right)+e^{-i \lambda \nu p} \delta\left(\boldsymbol{\kappa}+\boldsymbol{\kappa}_{0}\right)\right] \boldsymbol{Q}_{\lambda}\left(\boldsymbol{\kappa}_{0}\right)
$$

which satisfies (67). This is the Radon transform: $\boldsymbol{G}(p, \boldsymbol{\kappa})=\boldsymbol{F}^{\mathcal{R}}(p, \boldsymbol{\kappa})$ of the solution $\boldsymbol{F}(\boldsymbol{x})=e^{i \lambda \nu \boldsymbol{\kappa}_{0} \cdot \boldsymbol{x}} \boldsymbol{Q}_{\lambda}\left(\boldsymbol{\kappa}_{0}\right) \underline{\underline{6,10}}$

As another example, consider the Radon transform (59) of the L field. We can reproduce $\boldsymbol{G}(p, \boldsymbol{\kappa})=\boldsymbol{F}_{L}^{\mathcal{R}}(p, \boldsymbol{\kappa})$ from the first term in (69): $\boldsymbol{G}(p, \boldsymbol{\kappa})=\boldsymbol{\Gamma} \times\left(\Psi_{1} \boldsymbol{\omega}_{1}+\Psi_{2} \boldsymbol{\omega}_{2}\right)$ choosing $\Psi_{1}=\left(2 \pi i F_{0} / \nu^{3}\right) \delta\left(\kappa_{z}\right) e^{i \nu p}, \Psi_{2}=\left(2 \pi i F_{0} / \nu^{3}\right) \delta\left(\kappa_{z}\right) e^{-i \nu p}$ and $\boldsymbol{\omega}_{1}=\boldsymbol{L}, \boldsymbol{\omega}_{2}=\boldsymbol{L}^{\prime}$. The Radon transform of $J_{0}(\nu r) \boldsymbol{e}_{z}$ leads to a simpler choice: $\Psi=\left(2 \pi F_{0} / \nu^{3}\right) \delta\left(\kappa_{z}\right)\left(e^{i \nu p}+e^{-i \nu p}\right)$, $\boldsymbol{\omega}=\boldsymbol{e}_{z}$.

We can easily show that $\boldsymbol{G}(p, \boldsymbol{\kappa})$, (69) is an eigenvector

$$
R B S[\boldsymbol{G}(q, \boldsymbol{\kappa})](p, \boldsymbol{\kappa})=-\boldsymbol{\kappa} \times[\boldsymbol{\kappa} \times \boldsymbol{H}(p, \boldsymbol{\kappa})]=\frac{1}{\nu} \boldsymbol{G}(p, \boldsymbol{\kappa}),
$$

of the RBS operator (49), if $\boldsymbol{\omega}$ is a fixed vector: $\partial_{p} \boldsymbol{\omega}=0$. Here $\boldsymbol{H}(p, \boldsymbol{\kappa})=\Psi \boldsymbol{\omega}+(1 / \nu) \boldsymbol{\Gamma} \times(\Psi \boldsymbol{\omega})$ is a potential for $\boldsymbol{G}(p, \boldsymbol{\kappa}): \boldsymbol{G}(p, \boldsymbol{\kappa})=\boldsymbol{\Gamma} \times \boldsymbol{H}(p, \boldsymbol{\kappa})$ and we use (70). 
The CK method also enables us to write a simple integral expression for the solution of (67). We employ in (69) integral expressions for simple harmonic oscillations (70). If we assume $\Psi_{k}(p, \boldsymbol{\kappa}),(k=1,2)$ are separable in $p$ and $\boldsymbol{\kappa}$, these are given as

$$
\Psi_{k}(p, \kappa)=\frac{1}{4 \pi i \nu} U_{k}(\kappa) \int_{C_{\lambda}^{\mp}} \frac{e^{p \zeta}}{\zeta \mp i \lambda \nu} d \zeta
$$

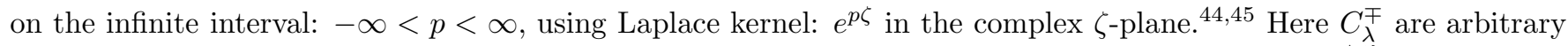
loops around the poles $\zeta= \pm i \lambda \nu$. This is basically the Cauchy integral representation formula for $e^{ \pm i \lambda \nu p}$. We find

$$
\boldsymbol{G}(p, \boldsymbol{\kappa})=\frac{1}{4 \pi i}\left[\left(i \lambda \boldsymbol{\kappa} \times \boldsymbol{\omega}_{1}-\boldsymbol{\kappa} \times \boldsymbol{\kappa} \times \boldsymbol{\omega}_{1}\right) \int_{C_{\lambda}^{-}} \frac{e^{p \zeta}}{\zeta-i \lambda \nu} d \zeta+\left(-i \lambda \boldsymbol{\kappa} \times \boldsymbol{\omega}_{2}-\boldsymbol{\kappa} \times \boldsymbol{\kappa} \times \boldsymbol{\omega}_{2}\right) \int_{C_{\lambda}^{+}} \frac{e^{p \zeta}}{\zeta+i \lambda \nu} d \zeta\right]
$$

using (73) in (69) and rearranging it. This is also oscillatory in $p$. We have included the $\boldsymbol{\kappa}$ dependent factors $U_{k}(\boldsymbol{\kappa})$ in the arbitrary functions $\boldsymbol{\omega}_{k}(\boldsymbol{\kappa})$. These are decoupled from the integral expressions.

For example, if we choose $\boldsymbol{\omega}_{1}(\boldsymbol{\kappa})=\left[(2 \pi)^{1 / 2} /\left(g \nu^{2}\right)\right] \boldsymbol{Q}_{\lambda}(\boldsymbol{\kappa}) s_{\lambda}(\lambda \nu \boldsymbol{\kappa})$ and $\boldsymbol{\omega}_{2}(\boldsymbol{\kappa})=\left[(2 \pi)^{1 / 2} /\left(g \nu^{2}\right)\right] \boldsymbol{Q}_{\lambda}(-\boldsymbol{\kappa}) s_{\lambda}(-\lambda \nu \boldsymbol{\kappa})$ in accordance with the Moses frame, we find $\boldsymbol{G}(p, \boldsymbol{\kappa})=\boldsymbol{F}^{\mathcal{R}}(p, \boldsymbol{\kappa})$, (19)).

We can reproduce the $\mathrm{L}$ field (59) choosing $\boldsymbol{\omega}_{1} \cong \delta\left(\kappa_{z}\right) \boldsymbol{e}_{z}$ and $\boldsymbol{\omega}_{2} \cong \delta\left(\kappa_{z}\right) \boldsymbol{e}_{z}$. We can also use $\boldsymbol{\omega}_{1} \cong \delta\left(\kappa_{z}\right) \boldsymbol{e}_{\psi}$ and $\boldsymbol{\omega}_{2} \cong \delta\left(\kappa_{z}\right) \boldsymbol{e}_{\alpha},\left(\kappa_{z}=\cos \alpha, \boldsymbol{\kappa}=\boldsymbol{e}_{r}\right)$ on $\mathbb{S}^{2}$.

If we substitute (74) in the inversion formula (A4), we find

$$
\begin{array}{r}
\boldsymbol{F}(\boldsymbol{x})=\frac{1}{32 \pi^{3} i} \nu^{2}\left[\int_{C_{\lambda}^{-}} \frac{1}{\zeta-i \lambda \nu} \int_{S^{2}}\left(i \lambda \boldsymbol{\kappa} \times \boldsymbol{\omega}_{1}-\boldsymbol{\kappa} \times \boldsymbol{\kappa} \times \boldsymbol{\omega}_{1}\right) e^{\zeta \boldsymbol{\kappa} \cdot \boldsymbol{x}} d \Omega d \zeta\right. \\
\left.+\int_{C_{\lambda}^{+}} \frac{1}{\zeta+i \lambda \nu} \int_{S^{2}}\left(-i \lambda \boldsymbol{\kappa} \times \boldsymbol{\omega}_{2}-\boldsymbol{\kappa} \times \boldsymbol{\kappa} \times \boldsymbol{\omega}_{2}\right) e^{\zeta \boldsymbol{\kappa} \cdot \boldsymbol{x}} d \Omega d \zeta\right]
\end{array}
$$

using the operator $\Gamma^{2}$ which is intertwined with $\nabla^{2}$, (68) and interchanging the order of integrations.

If we choose

$$
\begin{aligned}
& \boldsymbol{\omega}_{1}=-i(2 \pi)^{2} \frac{1}{\nu^{2}}\left[a \delta\left(\boldsymbol{\kappa}-\boldsymbol{\kappa}_{1}\right) \boldsymbol{E}_{1}+b \delta\left(\boldsymbol{\kappa}-\boldsymbol{\kappa}_{2}\right) \boldsymbol{E}_{2}+c \delta\left(\boldsymbol{\kappa}-\boldsymbol{\kappa}_{3}\right) \boldsymbol{E}_{3}\right] \\
& \boldsymbol{\omega}_{2}=-i(2 \pi)^{2} \frac{1}{\nu^{2}}\left[a \delta\left(\boldsymbol{\kappa}+\boldsymbol{\kappa}_{1}\right) \boldsymbol{E}_{1}+b \delta\left(\boldsymbol{\kappa}+\boldsymbol{\kappa}_{2}\right) \boldsymbol{E}_{2}+c \delta\left(\boldsymbol{\kappa}+\boldsymbol{\kappa}_{3}\right) \boldsymbol{E}_{3}\right],
\end{aligned}
$$

where $a, b, c$ are arbitrary constants, $i \lambda \boldsymbol{\kappa}_{i} \times \boldsymbol{E}_{i}=\boldsymbol{E}_{i}$ and $\boldsymbol{\kappa}_{i} \cdot \boldsymbol{E}_{i}=0$, the equation (75) yields

$$
\boldsymbol{F}(\boldsymbol{x})=-i\left(a e^{i \lambda \nu \boldsymbol{\kappa}_{1} \cdot \boldsymbol{x}} \boldsymbol{E}_{1}+b e^{i \lambda \nu \boldsymbol{\kappa}_{2} \cdot \boldsymbol{x}} \boldsymbol{E}_{2}+c e^{i \lambda \nu \boldsymbol{\kappa}_{3} \cdot \boldsymbol{x}} \boldsymbol{E}_{3}\right)
$$

The real part of this reduces to $a b c$ field $\underline{6}$ if we choose: $k_{1}=k_{2}=k_{3}=\lambda \nu, \boldsymbol{\kappa}_{1}=\boldsymbol{e}_{z}, \boldsymbol{\kappa}_{2}=\boldsymbol{e}_{x}, \boldsymbol{\kappa}_{3}=\boldsymbol{e}_{y}$ and $\boldsymbol{E}_{1}=\boldsymbol{e}_{x}+i \lambda \boldsymbol{e}_{y}, \boldsymbol{E}_{2}=\boldsymbol{e}_{y}+i \lambda \boldsymbol{e}_{z}, \boldsymbol{E}_{3}=\boldsymbol{e}_{z}+i \lambda \boldsymbol{e}_{x}$

We respectively refer the reader to Refs. 46, 47 for Fourier integral expression on $\mathbb{S}^{2}$ of the general solution to the scalar Helmholtz equation and to Refs. 48 -50 for integral representations of the Beltrami fields in physical space.

\section{TOPOLOGICALLY MASSIVE ABELIAN GAUGE FIELD}

The Euclidean topologically massive Abelian gauge field ${ }^{1-3}$ is a Trkalian field $\underline{6}^{6}$ Another example of Trkalian fields is the force-free magnetic field ${ }^{7}$ The Trkalian fields yield solutions of both the topologically massive gauge theories ${ }^{4}-6$ and gravity 20 The spherical curl and Radon transform analysis above are also valid for the Euclidean topologically massive Abelian gauge fields on $\mathbb{R}^{3}$. The gauge potential of the Trkalian field is also Trkalian. Meanwhile a gauge transformation corresponds to a vector normal to the sphere. The Radon transform of an anti-self-dual potential (or field) is related by antipodal map on the sphere to the transform of the self-dual potential obtained by inverting space coordinates.

The Trkalian fields L and CK are also solutions of this theory. Furthermore, the L solution provides a simple example for quantization of the topological mass in this context. 


\section{A. The gauge potential}

We can easily derive the spherical curl and Radon transforms of the potential following the same $\frac{10}{}$ reasoning above. We find

$$
\boldsymbol{A}_{\lambda}(\boldsymbol{x})=\frac{1}{g} \frac{1}{\nu} \int \chi_{\lambda}(\boldsymbol{x} \mid \lambda \nu \boldsymbol{\kappa}) s_{\lambda}(\lambda \nu \boldsymbol{\kappa}) d \Omega
$$

This satisfies the self-duality relation: $\boldsymbol{F}_{\lambda}=\nu \boldsymbol{A}_{\lambda}$ where $\boldsymbol{F}_{\lambda}=\boldsymbol{\nabla} \times \boldsymbol{A}_{\lambda}$, (16). We also find

$$
\boldsymbol{A}_{\lambda}^{\mathcal{R}}(p, \boldsymbol{\kappa})=(2 \pi)^{1 / 2} \frac{1}{g} \frac{1}{\nu^{3}}\left[e^{i \lambda \nu p} \boldsymbol{Q}_{\lambda}(\boldsymbol{\kappa}) s_{\lambda}(\lambda \nu \boldsymbol{\kappa})+e^{-i \lambda \nu p} \boldsymbol{Q}_{\lambda}(-\boldsymbol{\kappa}) s_{\lambda}(-\lambda \nu \boldsymbol{\kappa})\right]
$$

These only differ by a factor 6 of $1 / \nu$ from those expressions for $\boldsymbol{F}_{\lambda}$. Hence the potential $\boldsymbol{A}_{\lambda}^{\mathcal{R}}(p, \boldsymbol{\kappa}),(79)$ satisfies

$$
\boldsymbol{F}_{\lambda}^{\mathcal{R}}(p, \boldsymbol{\kappa})-\nu \boldsymbol{A}_{\lambda}^{\mathcal{R}}(p, \boldsymbol{\kappa})=0
$$

where $\boldsymbol{F}_{\lambda}^{\mathcal{R}}=\boldsymbol{\Gamma} \times \boldsymbol{A}_{\lambda}^{\mathcal{R}},(\underline{19})$.

A gauge transformation

$$
\boldsymbol{A}^{\prime}=\boldsymbol{A}-\frac{1}{g} \nabla U
$$

of the potential ${ }^{6}$ is given by a curl-free vector

$$
\nabla U(\boldsymbol{x})=\frac{1}{\nu} \int \chi_{0}(\boldsymbol{x} \mid \boldsymbol{k}) f_{0}(\boldsymbol{k}) d^{3} k
$$

This yields the gauge function

$$
U(\boldsymbol{x})=\frac{1}{(2 \pi)^{3 / 2}} i \frac{1}{\nu} \int e^{i \boldsymbol{k} \cdot \boldsymbol{x}} f_{0}(\boldsymbol{k}) \frac{1}{k} d^{3} k
$$

\section{B. The anti-self-dual case}

The anti-self-dual case

$$
\nabla \times \boldsymbol{F}+\nu \boldsymbol{F}=0,
$$

of equation (11) contains an extra factor of $(-)$ in $\nu$. This terminology is motivated by the interchange symmetry $\boldsymbol{F} \leftrightarrow \nu \boldsymbol{A}$. This leads to a flip of sign in the spherical curl and Radon transforms of the self-dual field (11). In this case, an arbitrary solution is given in terms of its transform on the sphere of radius $k=-\lambda \nu=|\nu|$. Furthermore, only the eigenfunctions for which $\lambda=-\operatorname{sgn}(\nu)$ contribute to the field. We find

$$
\begin{aligned}
\boldsymbol{F}_{\lambda}(\boldsymbol{x}) & =\frac{1}{g} \int \chi_{\lambda}(\boldsymbol{x} \mid-\lambda \nu \boldsymbol{\kappa}) s_{\lambda}(-\lambda \nu \boldsymbol{\kappa}) d \Omega \\
& =\frac{1}{(2 \pi)^{3 / 2}} \frac{1}{g} \int e^{-i \lambda \nu \boldsymbol{\kappa} \cdot \boldsymbol{x}} \boldsymbol{Q}_{\lambda}(\boldsymbol{\kappa}) s_{\lambda}(-\lambda \nu \boldsymbol{\kappa}) d \Omega
\end{aligned}
$$

and

$$
s_{\lambda}(-\lambda \nu \kappa)=\frac{1}{(2 \pi)^{1 / 2}} g \nu^{2} e^{i \lambda \nu p} F_{a}^{\mathcal{R}}(p, \kappa) .
$$


The Radon transform

$$
\boldsymbol{F}_{\lambda}^{\mathcal{R}}(p, \boldsymbol{\kappa})=(2 \pi)^{1 / 2} \frac{1}{g} \frac{1}{\nu^{2}}\left[e^{i \lambda \nu p} \boldsymbol{Q}_{\lambda}(-\boldsymbol{\kappa}) s_{\lambda}(\lambda \nu \boldsymbol{\kappa})+e^{-i \lambda \nu p} \boldsymbol{Q}_{\lambda}(\boldsymbol{\kappa}) s_{\lambda}(-\lambda \nu \boldsymbol{\kappa})\right]
$$

of the field satisfies: $\boldsymbol{\Gamma} \times \boldsymbol{F}_{\lambda}^{\mathcal{R}}=-\nu \boldsymbol{F}_{\lambda}^{\mathcal{R}}$. Noting $\lambda \nu=|\nu|>0$ for the self-dual case and $-\lambda \nu=|\nu|>0$ for the anti-self-dual case, the equation (87) coincides with (19). The difference of the two cases is the relative sign of $p$ and $\kappa$. We shall present a comparison of the two cases below.

The gauge potential

$$
\boldsymbol{A}_{\lambda}(\boldsymbol{x})=-\frac{1}{g} \frac{1}{\nu} \int \chi_{\lambda}(\boldsymbol{x} \mid-\lambda \nu \boldsymbol{\kappa}) s_{\lambda}(-\lambda \nu \boldsymbol{\kappa}) d \Omega
$$

for the field (85) satisfies the anti-self-duality relation: $\boldsymbol{F}_{\lambda}=-\nu \boldsymbol{A}_{\lambda}$. Its Radon transform

$$
\boldsymbol{A}_{\lambda}^{\mathcal{R}}(p, \boldsymbol{\kappa})=-(2 \pi)^{1 / 2} \frac{1}{g} \frac{1}{\nu^{3}}\left[e^{i \lambda \nu p} \boldsymbol{Q}_{\lambda}(-\boldsymbol{\kappa}) s_{\lambda}(\lambda \nu \boldsymbol{\kappa})+e^{-i \lambda \nu p} \boldsymbol{Q}_{\lambda}(\boldsymbol{\kappa}) s_{\lambda}(-\lambda \nu \boldsymbol{\kappa})\right]
$$

satisfies: $\boldsymbol{F}_{\lambda}^{\mathcal{R}}=-\nu \boldsymbol{A}_{\lambda}^{\mathcal{R}}$.

\section{The Radon transform and duality classes}

We can compare the Radon transforms of self-dual and anti-self-dual fields using a correspondence between them. A self-dual field becomes anti-self-dual, or vice versa under inversion of space coordinates, if the topological mass is held fixed $\underline{51}$ (Hence the topological mass is actually a pseudoscalar.) Therefore, given a self-dual-field $\boldsymbol{F}(\boldsymbol{x})$ : $\nabla_{x} \times \boldsymbol{F}(\boldsymbol{x})=\nu \boldsymbol{F}(\boldsymbol{x})$, we can write the corresponding anti-self-dual field as $\boldsymbol{F}^{\prime}(\boldsymbol{x})=\boldsymbol{F}(-\boldsymbol{x}): \nabla_{x} \times \boldsymbol{F}^{\prime}(\boldsymbol{x})=-\nu \boldsymbol{F}^{\prime}(\boldsymbol{x})$ inverting the coordinates: $\boldsymbol{x} \longrightarrow \boldsymbol{x}^{\prime}=-\boldsymbol{x}$.

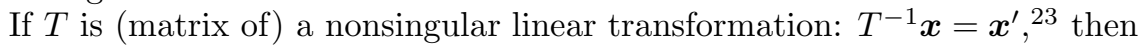

$$
\begin{aligned}
\boldsymbol{F}^{\prime} \mathcal{R}(p, \boldsymbol{\kappa}) & =\boldsymbol{\mathcal { R }}\left[\boldsymbol{F}^{\prime}(\boldsymbol{x})\right](p, \boldsymbol{\kappa})=\boldsymbol{\mathcal { R }}\left[\boldsymbol{F}\left(T^{-1} \boldsymbol{x}\right)\right](p, \boldsymbol{\kappa}) \\
& =\int \boldsymbol{F}\left(T^{-1} \boldsymbol{x}\right) \delta(p-\boldsymbol{\kappa} \cdot \boldsymbol{x}) d^{3} x \\
& =|T| \int \boldsymbol{F}\left(\boldsymbol{x}^{\prime}\right) \delta\left(p-T^{\dagger} \boldsymbol{\kappa} \cdot \boldsymbol{x}^{\prime}\right) d^{3} x^{\prime} \\
& =|T| \boldsymbol{F}^{\mathcal{R}}\left(p, T^{\dagger} \boldsymbol{\kappa}\right) .
\end{aligned}
$$

The inversion of coordinates is given by $T=-1,|T|=|\operatorname{det}[T]|=1$ which is an orthogonal matrix: $T T^{\dagger}=1$. Thus, the Radon transform of an anti-self-dual field

$$
\boldsymbol{F}^{\prime \mathcal{R}}(p, \boldsymbol{\kappa})=\boldsymbol{F}^{\mathcal{R}}(p,-\boldsymbol{\kappa}),
$$

is related by antipodal map on the sphere to the transform of the self-dual field obtained by inverting the coordinates.

In the Moses coframe, we can find the Radon transform of an anti-self-dual field similarly inverting the coordinates in (18). This leads to the inversion $\kappa \longrightarrow-\boldsymbol{\kappa}$ in (19). A flip of sign (21): $p \longrightarrow-p$ or $\boldsymbol{\kappa} \longrightarrow-\boldsymbol{\kappa}$ (but not both) brings $*{ }^{a} F^{R}(p,-\boldsymbol{\kappa})$ back to (19) with $\lambda \nu=|\nu|>0$ or (87) with $-\lambda \nu=|\nu|>0$. Note that the equation (91) together with (19) yields a representation different from (87) for anti-self-dual fields. These coincide upon both: $\nu \longrightarrow-\nu$ and $\kappa \longrightarrow-\kappa$.

The Radon transform of an anti-self-dual field or potential satisfy the equations (29) or (80) with an opposite sign for the topological mass as noted above. A simple example is given by $\boldsymbol{F}(\boldsymbol{x})=e^{i \mu \lambda \nu \boldsymbol{\kappa}_{0} \cdot \boldsymbol{x}} \boldsymbol{Q}_{\lambda}\left(\boldsymbol{\kappa}_{0}\right)$ where $\mu= \pm 1$ correspond to the self-dual and anti-self-dual cases. We find

$$
\boldsymbol{F}^{\mathcal{R}}(p, \boldsymbol{\kappa})=(2 \pi)^{2} \frac{1}{\nu^{2}}\left[e^{i \lambda \nu p} \delta\left(\boldsymbol{\kappa}-\mu \boldsymbol{\kappa}_{0}\right)+e^{-i \lambda \nu p} \delta\left(\boldsymbol{\kappa}+\mu \boldsymbol{\kappa}_{0}\right)\right] \boldsymbol{Q}_{\lambda}\left(\boldsymbol{\kappa}_{0}\right),
$$


using $\lambda \nu>0$ in the self-dual case (71) and $-\lambda \nu>0$ in the anti-self-dual case. This yields $\boldsymbol{\Gamma} \times \boldsymbol{F}^{\mathcal{R}}=\mu \nu \boldsymbol{F}^{\mathcal{R}}$ anticipating the effect of $\delta\left(\boldsymbol{\kappa} \mp \mu \boldsymbol{\kappa}_{0}\right) \underline{10}$

Noting the inversion of the refined transformation for a self-dual-field $\boldsymbol{F}^{R}(p, \boldsymbol{\kappa})$ on a canonical hemisphere in Subsection IIB 1, one can require $\boldsymbol{\kappa}$ to be restricted into the complementary hemisphere for the anti-self-dual field $\boldsymbol{F}^{\prime \mathcal{R}}(p, \boldsymbol{\kappa})$. (Also see Appendix A 1.)

\section{The Lundquist Solution}

The constant eigenvalue $\nu$, called the topological mass in this context, is intimately related to boundary conditions imposed on the field $19,36,37$ and the global topology determined by them. The quantization of the topological mass here arises as a result of a well defined gauge transformation as in the previous 4.6 examples.

The eigenvalue in $\mathrm{L}$ solution determines the twist per unit length of the field lines on the $z$-axis, see for example Refs. 52,53. In the topologically massive case, the strength of the field on the $z$-axis is proportional to the square of the eigenvalue. Meanwhile the gauge potential is of strength proportional to this twisting. This analogy suggests a natural geometric interpretation for the Lundquist field in this context.

We find the potential

$$
\boldsymbol{A}=\frac{1}{\nu} \boldsymbol{F}_{L}-\frac{1}{\nu} F_{0} \boldsymbol{e}_{z}
$$

integrating ${ }^{54}$ the components of $\boldsymbol{F}_{L}$, (55). This satisfies: $\boldsymbol{\nabla} \times \boldsymbol{A}-\nu \boldsymbol{A}=F_{0} \boldsymbol{e}_{z}$ with an extra term.

In the topologically massive Abelian gauge theory $\stackrel{4,6}{\underline{4}}$ we can make this term vanish: $\boldsymbol{F}_{L}^{\prime}-\nu \boldsymbol{A}^{\prime}=0$ by a gauge transformation: $\boldsymbol{A}^{\prime}=\boldsymbol{A}-(i / g) \boldsymbol{\nabla} \ln U$ identifying it with a gauge term: $-(i / g) \boldsymbol{\nabla} \ln U=(1 / \nu) F_{0} \boldsymbol{e}^{3}$. This yields $U=e^{i \nu z}$ which takes values in the group $U(1)$, choosing $F_{0}=\nu^{2} / g$. The strength of the gauge potential is given by the gauge coupling constant $\nu / g=n g$, if $\nu=n g^{2}$. This leads us to adopt a fundamental scale $\frac{4.6}{6}$ of length $l=2 \pi / g^{2}$. We can write $\nu=n g^{2}$ as $\nu=2 \pi n / l$. If $U=e^{i(2 \pi n / l) z}$ is a single-valued function of $z$ with the fundamental scale $l$, then $n$ has to be an integer. The fundamental length scale $l$ is the least common multiple of intervals over which the gauge function is single-valued and periodic for any integer $n$, in addition to the fact that it has a smaller period $l^{\prime}=l / n \underline{\text { 4.6 }}$ The discussion of physical and topological aspects of this solution and the effect of gauge transformations on these would be distracting us from our purposes here.

The anti-self-dual solution is given by $\kappa \longrightarrow-\kappa$ in (59). Also note that the $\mathrm{L}$ field defines a contact structure since $J_{0}$ and $J_{1}$ have no common zeros $\frac{55}{5}$

\section{CONCLUSION}

The spherical curl transformation for Trkalian fields is a Radon probe transformation in the Moses frame. We have written this using differential forms. This is an example of an integral transform which can be formally written this way.

The point of view here suggests an approach for studying the Trkalian fields in the transform space. The transform space representation has certain advantages. First of all, the equation in the transform space provides a concrete frame for studying these fields, for example using the spherical curl transform which is based on Moses eigenfunctions. This further offers a simplification since the differential and algebraic aspects are separable in the transform space. This also exhibits the role of the Moses eigenbasis which yields a helicity decomposition of the Radon transform. Meanwhile the field can be reconstructed using knowledge of the transform on a canonical hemisphere. Briefly, this equation provides a simple geometric frame in the transform space for Trkalian fields with a new insight into their structure besides practical advantages.

We have discussed the connection of the Radon transformation with the $B S$ integral. We have introduced the $R B S$ operator in the transform space. This is simply given in terms of 1-dimensional Fourier transforms. We can also associate an eigenvalue equation for $R B S$ operator with the Trkalian fields in transform space. The Radon transform of a Trkalian field is an eigenvector of this operator. Its kernel consists of those vector fields which are normal to the sphere. We have also presented an Ampere law type relation for these fields.

We have presented applications of these on the L solution. Then we have discussed the CK method with circular and elliptic solutions. The elliptic one reduces to VR solution. The L solution also defines a contact structure.

We have presented CK solution of the corresponding eigenvalue equation in the transform space. The CK method in transform space also leads to a simplification eliminating the differential operations and hence reducing the solution to simple algebraic manipulations consisting of arbitrary but fixed vectors. Because the Radon transform reduces the 
scalar Helmholtz equation to that of simple harmonic oscillations. The poloidal, toroidal representations also suggest the use of the Moses eigenbasis. This simplification also enables us to write integral representations as we have seen on a simple example. One can try imposing different boundary conditions and symmetries motivated by considerations in the physical space. The CK solution is also an eigenvector of the $R B S$ operator.

The Euclidean topologically massive Abelian gauge fields and also the force-free magnetic fields are examples of Trkalian fields. Hence the Radon and the spherical curl transform analysis are also valid for these. In the topologically massive case, the gauge potential is also Trkalian. The Radon transform of an anti-self-dual potential (or field) is related by antipodal map on the sphere to the transform of the self-dual potential obtained by inverting space coordinates.

The L and CK fields are also solutions of this theory. This has been overlooked previously. Furthermore, the L solution provides an example for quantization of the topological mass in this theory. This suggests a natural geometric interpretation for the Lundquist field in this context. However the discussion of physical implications of this is beyond our goals here.

\section{ACKNOWLEDGMENTS}

The author would like to thank the anonymous referee and the Editor for clear comments and guidance.

\section{APPENDIX A: THE RADON TRANSFORMATION}

\section{The Radon transformation}

The Radon transform of vector fields is defined ${ }^{10}$ as

$$
\boldsymbol{F}^{\mathcal{R}}(p, \boldsymbol{\kappa})=\boldsymbol{\mathcal { R }}[\boldsymbol{F}(\boldsymbol{x})](p, \boldsymbol{\kappa})=\int \boldsymbol{F}(\boldsymbol{x}) \delta(p-\boldsymbol{\kappa} \cdot \boldsymbol{x}) d^{3} x .
$$

This is a componentwise generalization of Radon transform

$$
f^{\mathcal{R}}(p, \boldsymbol{\kappa})=\boldsymbol{\mathcal { R }}[f(\boldsymbol{x})](p, \boldsymbol{\kappa}) \int f(\boldsymbol{x}) \delta(p-\boldsymbol{\kappa} \cdot \boldsymbol{x}) d^{3} x,
$$

of scalar functions in cartesian coordinates. This satisfies the identities

$$
f^{\mathcal{R}}(-p,-\boldsymbol{\kappa})=f^{\mathcal{R}}(p, \boldsymbol{\kappa}), \quad f^{\mathcal{R}}(-p, \boldsymbol{\kappa})=f^{\mathcal{R}}(p,-\boldsymbol{\kappa}) .
$$

The Radon transform (A1) of vectors also satisfies similar identities.

The Radon transform is simply defined ${ }^{23}, 26$ for (vector) functions in Schwartz class $\mathcal{S}\left[\mathbb{R}^{3}\right]$ of rapidly decreasing functions on $\mathbb{R}^{3}$. Hence the function vanishes at infinity as we assume repeatedly, for example in Sections $\amalg B$ and [II]. Then the Radon transform is a function in $\mathcal{S}\left[\mathbb{S}^{2} \times \mathbb{R}\right]$. A natural domain of definition for the Radon transform is the set of hyperplanes in $\mathbb{R}^{3}$, i. e. the projective space. The space $\mathbb{S}^{2} \times \mathbb{R}$ is a two-fold covering of this ${ }^{26,27,32}$ Note that the definition (A1) actually provides an extension 27,28 of the Radon transform as a function homogeneous of degree -1 on $\left(\mathbb{R}^{3}-\{0\}\right) \times \mathbb{R}$. The adjoint transform, see Definition 3 , works in the opposite direction. We refer the reader to Refs. 15, 26 28 or 25 for a derivation of (34). The inverse Radon transform is given as

$$
\boldsymbol{F}(\boldsymbol{x})=-\frac{1}{8 \pi^{2}} \nabla_{x}^{2} \int_{S^{2}} \boldsymbol{F}^{\mathcal{R}}(\boldsymbol{\kappa} \cdot \boldsymbol{x}, \boldsymbol{\kappa}) d \Omega
$$

where $\nabla_{x}^{2}$ is the Laplace operator.

We can prove the Fourier slice theorem (45) using (A1). The relation (46) for Fourier transform of the Riesz potential is valid for functions in the Schwartz class $\underline{27}$

The Radon probe transformation ${ }^{11}$ is defined as

$$
\mathfrak{R}[\boldsymbol{F}(\boldsymbol{x})](p, \boldsymbol{\kappa})=\boldsymbol{V}(p, \boldsymbol{\kappa}) \cdot \boldsymbol{\mathcal { R }}[\boldsymbol{F}(\boldsymbol{x})](p, \boldsymbol{\kappa}),
$$


where $\boldsymbol{V}(p, \boldsymbol{\kappa})$ is the probe.

Further note that, if we had defined the Radon transformation on the projective space of hyperplanes ${ }^{26}$ in $\mathbb{R}^{3}$, then in Section $\mathrm{V}$ we would take the Radon transforms of self-dual and anti-self-dual fields on distinct spaces.

\section{Inversion of the refined transformation}

The Radon transform (A1) is a right inverse: $\mathcal{R}_{H}^{-1} \mathcal{R}[\boldsymbol{F}](\boldsymbol{x})=\boldsymbol{F}(\boldsymbol{x})$ of the refined ${ }^{16}$ (inverse) transformation

$$
\begin{aligned}
\boldsymbol{\mathcal { R }}_{H}^{-1}\left[\boldsymbol{F}^{\mathcal{R}}(p, \boldsymbol{\kappa})\right](\boldsymbol{x}) & =-\frac{1}{4 \pi^{2}} \nabla^{2} \int_{H} \boldsymbol{F}^{\mathcal{R}}(\boldsymbol{\kappa} \cdot \boldsymbol{x}, \boldsymbol{\kappa}) d \Omega \\
& =-\frac{1}{4 \pi^{2}} \nabla^{2} \iint_{H} \boldsymbol{F}^{\mathcal{R}}(p, \boldsymbol{\kappa}) \delta(p-\boldsymbol{\kappa} \cdot \boldsymbol{x}) d \Omega d p,
\end{aligned}
$$

for any canonical hemisphere $H$. However as a left inverse, we find

$$
\begin{aligned}
\boldsymbol{R}_{H}^{-1}\left[\boldsymbol{F}\left(p^{\prime}, \boldsymbol{\kappa}^{\prime}\right)\right](p, \boldsymbol{\kappa}) & =-\iint_{H} \boldsymbol{F}\left(p^{\prime}, \boldsymbol{\kappa}^{\prime}\right) W\left(p, \boldsymbol{\kappa} ; p^{\prime}, \boldsymbol{\kappa}^{\prime}\right) d \Omega^{\prime} d p^{\prime} \\
& =\left\{\begin{array}{l}
\boldsymbol{F}(p, \boldsymbol{\kappa}), \boldsymbol{\kappa} \in H, \\
\boldsymbol{F}(-p,-\boldsymbol{\kappa}), \boldsymbol{\kappa} \in H^{\prime}
\end{array}\right.
\end{aligned}
$$

where

$$
\begin{aligned}
W\left(p, \boldsymbol{\kappa} ; p^{\prime}, \boldsymbol{\kappa}^{\prime}\right) & =\frac{1}{(2 \pi)^{2}} \int \delta(p-\boldsymbol{\kappa} \cdot \boldsymbol{x}) \nabla_{x}^{2} \delta\left(p^{\prime}-\boldsymbol{\kappa}^{\prime} \cdot \boldsymbol{x}\right) d^{3} x \\
& =-\delta\left(p^{\prime}-p\right) \delta\left(\boldsymbol{\kappa}^{\prime}-\boldsymbol{\kappa}\right)-\delta\left(p^{\prime}+p\right) \delta\left(\boldsymbol{\kappa}^{\prime}+\boldsymbol{\kappa}\right) .
\end{aligned}
$$

We refer the reader to Ref. 16 for evaluation of this integral. Thus only in the case $\boldsymbol{F}(p, \boldsymbol{\kappa})$ is the Radon transform of a function can we define both right and left inverse of this refined transformation, $\underline{16}$

\section{APPENDIX B: THE SPHERICAL CURL AND RADON TRANSFORMS}

The inverse Radon transform in differential forms is given as

$$
*{ }^{a} F(\boldsymbol{x})=\frac{1}{8 \pi^{2}} \triangle_{x} \int_{S^{2}} *{ }^{a} F^{\mathcal{R}}(\boldsymbol{\kappa} \cdot \boldsymbol{x}, \boldsymbol{\kappa}) d \Omega,
$$

where $\triangle_{x}=(d+\delta)^{2}=\delta d+d \delta$ is the Laplace-Beltrami operator and $\delta \omega_{r}=(-1)^{r} * d * \omega_{r}$ for an $r$-form $\omega_{r}$.

We can prove equivalence of the spherical curl transform and the Radon transform as follows $\frac{10}{\underline{10}}$ If we substitute (17) in (16), we find

$$
*^{a} F(\boldsymbol{x})=\frac{1}{(2 \pi)^{2}} \nu^{2} \int e^{-i \lambda \nu(p-\boldsymbol{\kappa} \cdot \boldsymbol{x})} \omega^{a}(\boldsymbol{\kappa}) F_{a}^{\mathcal{R}}(p, \boldsymbol{\kappa}) d \Omega
$$

We can write this as

$$
{ }^{a} F(\boldsymbol{x})=\frac{1}{(2 \pi)^{2}} \nu^{2} d x^{i} \int e^{-i \lambda \nu(p-\boldsymbol{\kappa} \cdot \boldsymbol{x})} \mid \omega_{i}^{a}(\boldsymbol{\kappa})><\omega_{k}^{a}(\boldsymbol{\kappa}), *^{a} \mathcal{F}_{k}^{\mathcal{R}}(p, \boldsymbol{\kappa})>d \Omega
$$

We find

$$
\int e^{-i \lambda \nu(p-\boldsymbol{\kappa} \cdot \boldsymbol{x})}\left|\omega_{i}^{a}(\boldsymbol{\kappa})><\omega_{k}^{a}(\boldsymbol{\kappa}), *^{a} \mathcal{F}_{k}^{\mathcal{R}}(p, \boldsymbol{\kappa})>d \Omega=\frac{1}{2} \int e^{-i \lambda \nu(p-\boldsymbol{\kappa} \cdot \boldsymbol{x})} \delta_{i k}\right| *{ }^{a} \mathcal{F}_{k}^{\mathcal{R}}(p, \boldsymbol{\kappa})>d \Omega,
$$


following a similar reasoning given in Ref. 10 with our notation. Here we use the completeness relation (5) for the coframe, the expression (19) for the Radon transform of the field which yields $\left\langle\omega^{3}, *^{a} \mathcal{F}^{\mathcal{R}}\right\rangle=0$ and the fact that the integrals of the two remaining terms over the sphere are equal. Then we are led to

$$
*^{a} F(\boldsymbol{x})=\frac{1}{2(2 \pi)^{2}} \nu^{2} \int e^{-i \lambda \nu(p-\boldsymbol{\kappa} \cdot \boldsymbol{x})} *{ }^{a} F^{\mathcal{R}}(p, \boldsymbol{\kappa}) d \Omega .
$$

One recovers the inverse Radon transform formula (B1) introducing the Laplace-Beltrami operator and restricting $p=\boldsymbol{\kappa} \cdot \boldsymbol{x} \cdot \underline{\underline{10}}$

Also substituting (19) for $p=\boldsymbol{\kappa} \cdot \boldsymbol{x}$ in (B1)

$$
{ }^{a} F(\boldsymbol{x})=\frac{1}{2(2 \pi)^{3 / 2}} \frac{1}{g} \frac{1}{\nu^{2}} \triangle_{x} \int\left[e^{i \lambda \nu \boldsymbol{\kappa} \cdot \boldsymbol{x}} \omega^{a}(\boldsymbol{\kappa}) s_{a}(\lambda \nu \boldsymbol{\kappa})+e^{-i \lambda \nu \boldsymbol{\kappa} \cdot \boldsymbol{x}} \omega^{a}(-\boldsymbol{\kappa}) s_{a}(-\lambda \nu \boldsymbol{\kappa})\right] d \Omega,
$$

we see that the inverse Radon transform (B1) yields the expression (16) of $*^{a} F(\boldsymbol{x})$ in terms of the spherical curl transform $\stackrel{10}{\underline{10}}$ Here the second integral reduces to the first one as $\kappa \longrightarrow-\kappa$.

\section{APPENDIX C: BIOT-SAVART INTEGRAL OPERATOR}

i) $\boldsymbol{\nabla}_{x} \cdot B S[\boldsymbol{F}](\boldsymbol{x})=0$,

$$
\begin{aligned}
\boldsymbol{\nabla} \cdot B S[\boldsymbol{F}](\boldsymbol{x}) & =\boldsymbol{\nabla} \cdot \boldsymbol{\nabla} \times A[\boldsymbol{F}](\boldsymbol{x}) \\
& =\frac{1}{4 \pi} \boldsymbol{\nabla} \cdot \int_{D} \boldsymbol{F}(\boldsymbol{y}) \times \frac{\boldsymbol{x}-\boldsymbol{y}}{|\boldsymbol{x}-\boldsymbol{y}|^{3}} d^{3} y \\
& =0 .
\end{aligned}
$$

ii) $\boldsymbol{\nabla}_{x} \times B S[\boldsymbol{F}](\boldsymbol{x})=\boldsymbol{F}(\boldsymbol{x})$,

$$
\begin{aligned}
\boldsymbol{\nabla}_{x} \times B S[\boldsymbol{F}](\boldsymbol{x}) & =\boldsymbol{\nabla}_{x} \times \boldsymbol{\nabla}_{x} \times A[\boldsymbol{F}](\boldsymbol{x}) \\
& =\frac{1}{4 \pi} \boldsymbol{\nabla}_{x} \times \boldsymbol{\nabla}_{x} \times \int_{D} \frac{\boldsymbol{F}(\boldsymbol{y})}{|\boldsymbol{x}-\boldsymbol{y}|} d^{3} y \\
& =-\frac{1}{4 \pi} \nabla_{x}^{2} \int_{D} \frac{\boldsymbol{F}(\boldsymbol{y})}{|\boldsymbol{x}-\boldsymbol{y}|} d^{3} y+\frac{1}{4 \pi} \boldsymbol{\nabla}_{x} \int_{D} \boldsymbol{F}(\boldsymbol{y}) \cdot \boldsymbol{\nabla}_{x} \frac{1}{|\boldsymbol{x}-\boldsymbol{y}|} d^{3} y \\
& =-\frac{1}{4 \pi} \int_{D} \boldsymbol{F}(\boldsymbol{y}) \nabla^{2} \frac{1}{|\boldsymbol{x}-\boldsymbol{y}|} d^{3} y-\frac{1}{4 \pi} \boldsymbol{\nabla}_{x} \int_{D} \boldsymbol{F}(\boldsymbol{y}) \cdot \nabla_{y} \frac{1}{|\boldsymbol{x}-\boldsymbol{y}|} d^{3} y .
\end{aligned}
$$

We can write the second integral as

$$
\begin{aligned}
\int_{D} \boldsymbol{F}(\boldsymbol{y}) \cdot \nabla_{y} \frac{1}{|\boldsymbol{x}-\boldsymbol{y}|} d^{3} y & =\int_{D} \boldsymbol{\nabla}_{y} \cdot\left[\frac{\boldsymbol{F}(\boldsymbol{y})}{|\boldsymbol{x}-\boldsymbol{y}|}\right] d^{3} y-\int_{D} \frac{\boldsymbol{\nabla}_{y} \cdot \boldsymbol{F}(\boldsymbol{y})}{|\boldsymbol{x}-\boldsymbol{y}|} d^{3} y \\
& =-\int_{D} \frac{\boldsymbol{\nabla}_{y} \cdot \boldsymbol{F}(\boldsymbol{y})}{|\boldsymbol{x}-\boldsymbol{y}|} d^{3} y+\oint_{S} \frac{\boldsymbol{F}(\boldsymbol{y})}{|\boldsymbol{x}-\boldsymbol{y}|} \cdot d \boldsymbol{s} .
\end{aligned}
$$

This yields

$$
\begin{aligned}
\boldsymbol{\nabla}_{x} \times B S[\boldsymbol{F}](\boldsymbol{x}) & =\boldsymbol{F}(\boldsymbol{x})+\frac{1}{4 \pi} \nabla_{x} \int_{D} \frac{\boldsymbol{\nabla}_{y} \cdot \boldsymbol{F}(\boldsymbol{y})}{|\boldsymbol{x}-\boldsymbol{y}|} d^{3} y-\frac{1}{4 \pi} \nabla_{x} \oint_{S} \frac{\boldsymbol{F}(\boldsymbol{y})}{|\boldsymbol{x}-\boldsymbol{y}|} \cdot d \boldsymbol{s}_{y} \\
& =\boldsymbol{F}(\boldsymbol{x})-\frac{1}{4 \pi} \int_{D} \boldsymbol{\nabla}_{y} \cdot \boldsymbol{F}(\boldsymbol{y}) \frac{\boldsymbol{x}-\boldsymbol{y}}{|\boldsymbol{x}-\boldsymbol{y}|^{3}} d^{3} y+\frac{1}{4 \pi} \oint_{S} \frac{\boldsymbol{x}-\boldsymbol{y}}{|\boldsymbol{x}-\boldsymbol{y}|^{3}} \boldsymbol{F}(\boldsymbol{y}) \cdot d \boldsymbol{s}_{y},
\end{aligned}
$$


for $\boldsymbol{x} \in D$. Thus we conclude

$$
\boldsymbol{\nabla}_{x} \times B S[\boldsymbol{F}](\boldsymbol{x})=\boldsymbol{F}(\boldsymbol{x}), \quad \boldsymbol{x} \in D,
$$

if

a) $\boldsymbol{F}(\boldsymbol{y})$ is divergence-free: $\boldsymbol{\nabla}_{y} \cdot \boldsymbol{F}(\boldsymbol{y})=0$,

b) $\boldsymbol{F}(\boldsymbol{y})$ vanishes at infinity, as the region $D$ is extended to whole space $\mathbb{R}^{3}$ [for a finite region $D, \boldsymbol{F}(\boldsymbol{y})$ is tangent to the surface $S=\partial D$ bounding this region].

\section{APPENDIX D: BIOT-SAVART INTEGRAL OF THE LUNDQUIST FIELD}

We shall evaluate

$$
\begin{aligned}
B S\left[\boldsymbol{F}_{L}\right](\boldsymbol{x}) & =\frac{1}{4 \pi} \int_{D} \boldsymbol{F}_{L}(\boldsymbol{y}) \times \frac{\boldsymbol{x}-\boldsymbol{y}}{|\boldsymbol{x}-\boldsymbol{y}|^{3}} d^{3} y \\
& =I_{1}+I_{2}+I_{3}+I_{4}+I_{5},
\end{aligned}
$$

which can be decomposed into five terms. We choose: $\boldsymbol{y}=r \boldsymbol{e}_{r}(\phi)+z \boldsymbol{e}_{z}$ and $\boldsymbol{x}=\boldsymbol{R e}_{r}(\theta)$ since $\boldsymbol{F}_{L}$ is invariant along the $z$-axis. We shall need

$$
\int_{z=-\infty}^{\infty} \frac{z}{\left(a^{2}+z^{2}\right)^{3 / 2}} d z=0, \quad \int_{z=-\infty}^{\infty} \frac{1}{\left(a^{2}+z^{2}\right)^{3 / 2}} d z=2 \frac{1}{a^{2}} .
$$

We shall also make use of Poisson integral formulas

$$
\begin{aligned}
& \frac{1}{2 \pi} \int_{\phi=0}^{2 \pi} P(R, r, \phi-\theta) d \phi=1, \quad \frac{1}{2 \pi} \int_{\phi=0}^{2 \pi} R \sin \phi P(R, r, \phi-\theta) d \phi=r \sin \theta \\
& \frac{1}{2 \pi} \int_{\phi=0}^{2 \pi} R \cos \phi P(R, r, \phi-\theta) d \phi=r \cos \theta,
\end{aligned}
$$

for the region $r \leq R$. Here $P(R, r, \phi-\theta)$ is the Poisson kernel

$$
P(R, r, \phi-\theta)=\frac{R^{2}-r^{2}}{R^{2}+r^{2}-2 r R \cos (\phi-\theta)} .
$$

We interchange $R$ and $r$ in (D3) for the region $R<r$. One can easily prove these, for example following Ref. 56. For each term in (D1), we shall first carry out integration over $z$ and decompose the resulting integral into two pieces corresponding to regions $r \leq R$ and $R<r$. Then we shall carry out integrations over $\phi$ using (D3). We shall end up with integrals over $r(x=\nu r)$ combining these again. The first integral

$$
\begin{aligned}
I_{1} & =-\frac{1}{4 \pi} \int \frac{z F_{\phi}(r) \boldsymbol{e}_{r}(\phi)}{\left[R^{2}+r^{2}-2 r R \cos (\phi-\theta)+z^{2}\right]^{3 / 2}} r d r d \phi d z, \\
& =0
\end{aligned}
$$

immediately vanishes upon integration over $z$. The second term yields

$$
\begin{aligned}
I_{2} & =\frac{1}{4 \pi} \int \frac{R F_{z}(r) \boldsymbol{e}_{\theta}}{\left[R^{2}+r^{2}-2 r R \cos (\phi-\theta)+z^{2}\right]^{3 / 2}} r d r d \phi d z \\
& =\frac{1}{\nu} F_{0}\left[X \int_{x=0}^{X} \frac{J_{0}(x)}{X^{2}-x^{2}} x d x+X \int_{x=X}^{\infty} \frac{J_{0}(x)}{x^{2}-X^{2}} x d x\right] \boldsymbol{e}_{\theta} .
\end{aligned}
$$

The third integral is 


$$
\begin{aligned}
I_{3} & =-\frac{1}{4 \pi} \int \frac{r F_{z}(r) \boldsymbol{e}_{\phi}}{\left[R^{2}+r^{2}-2 r R \cos (\phi-\theta)+z^{2}\right]^{3 / 2}} r d r d \phi d z \\
& =\frac{1}{\nu} F_{0} \frac{1}{X} \int_{x=0}^{X} J_{0}(x) x d x \boldsymbol{e}_{\theta}-I_{2} .
\end{aligned}
$$

The fourth integral yields

$$
\begin{aligned}
I_{4} & =-\frac{1}{4 \pi} \int \frac{R F_{\phi}(r) \cos (\phi-\theta) \boldsymbol{e}_{z}}{\left[R^{2}+r^{2}-2 r R \cos (\phi-\theta)+z^{2}\right]^{3 / 2}} r d r d \phi d z \\
& =-\frac{1}{\nu} F_{0}\left[\int_{x=0}^{X} \frac{J_{1}(x)}{X^{2}-x^{2}} x^{2} d x+X^{2} \int_{x=X}^{\infty} \frac{J_{1}(x)}{x^{2}-X^{2}} d x\right] \boldsymbol{e}_{z}
\end{aligned}
$$

The fifth integral is

$$
\begin{aligned}
I_{5} & =\frac{1}{4 \pi} \int \frac{r F_{\phi}(r) \boldsymbol{e}_{z}}{\left[R^{2}+r^{2}-2 r R \cos (\phi-\theta)+z^{2}\right]^{3 / 2}} r d r d \phi d z \\
& =\frac{1}{\nu} F_{0} \int_{x=X}^{\infty} J_{1}(x) d x \boldsymbol{e}_{z}-I_{4} .
\end{aligned}
$$

Thus we find

$$
\begin{aligned}
B S\left[\boldsymbol{F}_{L}\right](\boldsymbol{x}) & =\frac{1}{\nu} F_{0}\left[\frac{1}{X} \int_{x=0}^{X} J_{0}(x) x d x \boldsymbol{e}_{\theta}+\int_{x=X}^{\infty} J_{1}(x) d x \boldsymbol{e}_{z}\right] \\
& =\frac{1}{\nu} \boldsymbol{F}_{L}(\boldsymbol{x}),
\end{aligned}
$$

adding up these terms. Here, we use

$$
\frac{1}{X} \int_{x=0}^{X} J_{0}(x) x d x=J_{1}(X), \quad \int_{x=X}^{\infty} J_{1}(x) d x=J_{0}(X) .
$$

1 S. Deser, R. Jackiw, S. Templeton, Phys. Rev. Lett. 48, 975 (1982).

2 S. Deser, R. Jackiw, S. Templeton, Ann. Phys. 140, 372 (1982).

3 J. F. Schonfeld, Nuc. Phys. B 185, 157 (1981).

${ }^{4}$ K. Saygili, arXiv: hep-th/0610307.

5 K. Saygili, Int. J. Mod. Phys. A 22, 2961 (2007), arXiv: hep-th/0611046

${ }^{6}$ K. Saygili, Int. J. Mod. Phys. A 23, 2015 (2008), arXiv: hep-th/0711.3893.

7 G. E. Marsh, Force-free Magnetic Fields, Solutions, Topology and Applications (World Scientific, 1996).

${ }^{8}$ H. E. Moses, Siam J. Appl. Math. 21, 114 (1971).

9 M. A. MacLeod, J. Math. Phys. 36, 2951 (1995).

10 M. A. MacLeod, J. Math. Phys. 39, 1642 (1998).

11 F. Natterer, F. Wübbeling, Mathematical Methods in Image Reconstruction (SIAM, 2001).

12 P. Hillion, Adv. Appl. Clifford Alg. 4, 13 (1994).

13 P. Hillion, A. Lakhtakia, Phil. Trans. Roy. Soc. London Series A 344, 235 (1993).

14 I. M. Gelfand, S. G. Gindikin, M. I. Graev, Selected Topics in Integral Geometry, Translations of Mathematical Monographs Vol. 220 (American Mathematical Society, 2003).

15 V. Palamodov, Reconstructive Integral Geometry (Birkhäuser, 2004).

16 H. E. Moses, R. T. Prosser, Proc. R. Soc. Lond. A 422, 343 (1989).

17 J. Cantarella, D. DeTurck, H. Gluck, Jour. Math. Phys. 42, 876 (2001).

18 S. Lundquist, Arkiv för Fysik 2, 361 (1950).

19 S. Chandrasekhar, P. C. Kendall, Astrophys. Jour. 126, 457 (1957). 
20 A. Aliev, Y. Nutku, K. Saygili, Class. \& Quan. Grav. 17, 4111 (2000).

21 M. Dahl, Contact and Symplectic Geometry in Electromagnetism, M. Sc. Thesis, Helsinki University of Technology (2002), url http://users.math.tkk.fi/ fdahl/casgiem.pdf.

${ }^{22}$ M. Dahl, Contact Structures on $\mathbb{R}^{3}$ from Trkalian Fields and Maxwell's Equations, preprint, url http://users.math.tkk.fi/$\sim$ fdahl/beltrami.pdf.

${ }^{23}$ I. M. Gel'fand, M. I. Graev, N. Ya. Vilenkin, Generalized Functions Volume 5, Integral Geometry and Representation Theory (Academic Press, 1966).

24 C. A. Berenstein, Radon transforms, wavelets, and applications in Integral Geometry, Radon Transforms and Complex Analysis C. A. Berenstein, P. F. Ebenfelt, S. G. Gindikin, S. Helgason, A. E. Tumanov, eds. E. Casadio Tarabusi, M. A. Picardello, G. Zampieri, Lecture Notes in Mathematics 1684 (Springer, 1998).

25 S. R. Deans, The Radon Transform and Some of Its Applications (Dover, 2007).

26 S. Helgason, The Radon Transform (Birkhäuser, 1999).

27 A. Markoe, Analytic Tomography, Encyclopedia of Mathematics and Its Applications Vol. 106 (Cambridge University Press, 2006).

${ }^{28}$ F. Natterer, The Mathematics of Computerized Tomography (SIAM, 2001).

29 R. J. Parsley, The Biot-Savart Operator and Electrodynamics on Bounded Subdomains of the Three-sphere, Ph. D. Thesis, University of Pennsylvania (2004), url http://www.wfu.edu/ parslerj/research/dissertation.parsley.pdf

30 J. Cantarella, D. DeTurck, H. Gluck, M. Teytel, Jour. Math. Phys. 41, 5615 (2000).

31 J. Cantarella, D. DeTurck, H. Gluck, M. Teytel, Phys. Plasmas 7, 2766 (2000).

32 A. G. Ramm, A. I. Katsevich, The Radon Transform and Local Tomography (CRC Press, 1996).

33 G. E. Marsh, Phys. Rev. A 45, 7520 (1992).

${ }^{34}$ O. Bjørgum, Univ. Bergen Arbok Naturvitensk. Rekke 1, 1 (1951).

35 O. Bjørgum, T.Godal, Univ. Bergen Arbok Naturvitensk. Rekke 13, 1 (1952).

36 R. Lüst, A. Schlüter, Z. Astrophys. 34, 263 (1954).

37 S. Chandrasekhar, Proc. Nat. Acad. Sci. 42, 1 (1956).

38 S. Chandrasekhar, Astrophys. Jour. 124, 232 (1956).

39 G. F. Torres del Castillo, 3-D Spinors, Spin-Weighted Functions and Their Applications (Birkhäuser, 2003).

40 Z. Yoshida, J. Math. Phys. 33, 1252 (1992).

41 G. F. Torres del Castillo, J. Math. Phys. 35, 499 (1994).

42 M. Vandas, E. P. Romashets, A\&A 398, 801 (2003).

43 M. Vandas, E. P. Romashets, Magnetic Field in an Elliptic Flux Rope: A Generalization of the Lundquist Solution in Proc. 10th European Solar Physics Meeting Solar Variability: From Core to Outer Frontiers, ed. A. Wilson, ESA SP-506 Vol. 1 (ESA Publications Division, 2002).

44 E. L. Ince, Ordinary Differential Equations (Dover, 1956).

45 D. Zwillinger, Handbook of Differential Equations (Academic Press, 1989).

46 W. Miller, Jr., Symmetry and Separation of Variables, Encyclopedia of Mathematics and Its Applications Vol. 4 (AddisonWesley, 1977).

47 R. G. Newton, J. Math. Phys. 33, 44 (1992).

48 V. V. Kravchenko, J. Phys. A: Math. Gen. 36, 1515 (2003).

49 V. V. Kravchenko, M. V. Shapiro, Integral representations for spatial models of mathematical physics, Pitman Res. Notes in Math. Series Vol. 351 (Addison Wesley Longman, 1996).

50 A. Lakhtakia, Beltrami Fields in Chiral Media (World Scientific, 1994).

51 K. R. Brownstein, Phys. Rev. A 35, 4856 (1987).

52 P. M. Bellan, J. Yee, J. F. Hansen, Earth Planets Space 53, 495 (2001).

53 S. Dasso, C. H. Mandrini, P. Demoulin, M. L. Luoni, A\&A 455, 349 (2006).

${ }^{54}$ H. Goedbloed, S. Poedts, Principles of Magnetohydrodynamics with Applications to Laboratory and Astrophysical Plasmas (Cambridge University Press, 2004).

55 A. Gray, G. B. Mathews, A Treatise on Bessel Functions and Their Applications to Physics (Dover, 1966).

56 A. Jeffrey, Applied Partial Differential Equations, An Introduction (Academic Press, 2003). 